\title{
I sottogruppi del gruppo modulare con coefficienti del corpo di Jacobi-Eisenstein e un teorema sui gruppi finiti.
}

\author{
Memoria di Giovanni Sansone (a Firenza).
}

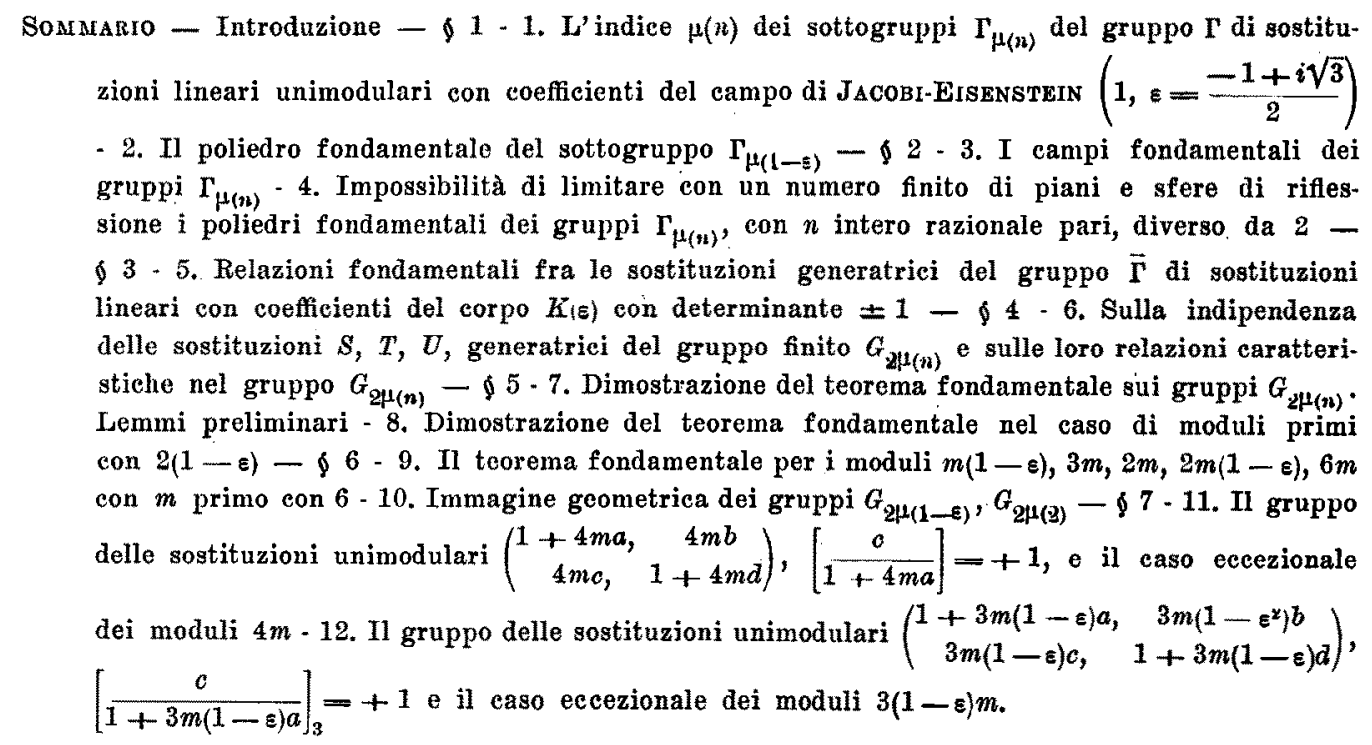

\section{INTRODUZIONE}

Nella mia Memoria I sotlogruppi del gruppo di Picard e due teoremi sui gruppi finiti analoghi al teorema del Dick $\left(^{1}\right)$ e nella Nota Le relazioni fondamentali tra le operazioni generatrici del gruppo modulare finito con coefficienti del campo di Gauss $\left({ }^{2}\right)$ scrissi che i metodi da me trovati potevano applicarsi allo studio dei gruppi del BraNchr di sostituzioni lineari con

(1) Rendiconti del Circolo Matematico di Palermo (Tomo XLVII, pp. 278-332). Nol seguito le citazioni relative a questa Memoria saranno indicate con (A).

(*) Rendiconti del Circolo Matematico di Palermo, Tomo XLIX. 
coefficienti appartenenti a corpi quadratici immaginari ( $\left.{ }^{3}\right)$. In questa Nota infatti io studio i gruppi $\Gamma$ di sostituzioni unimodulari

$$
z^{\prime}=\frac{\alpha z+\beta}{\gamma \tilde{s}+\delta}, \quad \alpha \delta-\beta \gamma=1
$$

con coefficienti $\alpha, \beta, \gamma, \delta$ interi del corpo $K(\varepsilon),\left(\varepsilon=\frac{-1+i \sqrt{3}}{2}\right)$ di $J_{\text {ACOBI- }}$ Ersenstrern delle radici cubiche dell' unita, ma le ricerche possono estendersi ai gruppi di sostituzioni lineari unimodulari con coefficienti dei corpi $K(i \sqrt{2})$; $K\left(\frac{-1+i \sqrt{D}}{2}\right), D=7,11,15,19 \mathrm{e}$ in generale a quei corpi il cui poliedro fondamentale abbia un solo vertice singolare $\left({ }^{4}\right)$ e si possa limitare con un numero finito di piani e sfere di riflessioni.

Il procedimento generale che si presenta è il seguente. Dato il gruppo $\Gamma$ di sostituzioni lineari unimodulari

$$
z^{\prime}=\frac{\alpha z+\beta}{\gamma z+\delta}, \quad \alpha \delta-\beta \gamma=1
$$

con coefficienti di un corpo quadratico immaginario, si può determinare con un semplice ragionamento aritmetico l'indice finito $\mu(n)$ del suo sottogruppo invariante $\Gamma_{\mu(n)}$ formato dalle sostituzioni $\left(\begin{array}{l}\alpha, \beta \\ \gamma, \delta\end{array}\right)$ caratterizzate dalle relazioni:

$$
\left(\begin{array}{ll}
\alpha, & \beta \\
\gamma, \delta
\end{array}\right) \equiv\left(\begin{array}{ll}
1, & 0 \\
0, & 1
\end{array}\right)
$$

(mod. $n)$,

con $n$ intero del corpo ( $\$ 1$, (A)). Costruito il poliedro fondamentale del gruppo $\Gamma$, si determinano, combinando le riflessioni sulle facce del poliedro, le sue sostituzioni ellittiche generatrici $(\S 5,(A))$; queste sono anche le sostituzioni ellittiche generatrici del gruppo modulare finito $G_{\mu(n)}$ di ordine $\mu(n)$ mediante il quale $\Gamma$ si rappresenta per $\Gamma_{\mu(n)}$. Un primo gruppo di relazioni tra le sostituzioni di $G_{\mu(n)}$ sono quelle che esprimono il periodo delle sue sostituzioni ellittiche generatrici, un altro gruppo di condizioni si ottiene

(3) L. BranchI: Sui gruppi di sostituzioni lineari appartenenti a corpi quadratici immaginari. (Matematiache Annalen, Bd. XI, pp. 332-412).

(4) Questa circostanza si deve presentare secondo un teorema enunciato dal BraNOHI, ma ancora non dimostrato, per i corpi quadratici privi di illeali secondari. Cfr. I. BrancHI, loc. cit. $\left({ }^{3}\right)$, p. 383 . 
dall' esprimere che le sostituzioni paraboliche del gruppo $\Gamma_{\mu(n)}$ si rappresentano in $G_{\mu(n)}$ con l'unità (\$ 7, (A)). Per provare poi se le relazioni trovate tra le sustituzioni generatrici di $G_{\mu(n)}$ sono sufficienti a caratterizzarlo, basta dimostrare che le sostituzioni unimodulari $\left(\begin{array}{cc}1+n a, & n b \\ n c, & 1+n d\end{array}\right)$ con $a, b, c, d$ interi del corpo sono esprimibili come prodotti di un numero finito di sostituzioni paraboliche di $\Gamma_{\mu(n)}(\$ 8$ a $\$ 11,(\mathrm{~A}))$. Questa proposizione non è in generale vera nel corpo di GaUss $K(i)$, ove fanno eccezione i moduli $n \equiv 0$ (mod. 4) $\left(^{5}\right)$, cosi nel corpo $K(\varepsilon)$ fanno soltanto eccezione i moduli divisibili per $2^{\lambda},(1-\varepsilon)^{\lambda+1}$ con $\lambda>2$, in altre parole quei moduli che contengono il divisore $2^{2}$, o la potenza $(1-\varepsilon)^{3}$ del divisore $1-\varepsilon$ del numero primo critico del corpo.'

Riassumiamo ora i resultati di questa Nota.

L'indice $\mu(n)$ del gruppo $\Gamma_{\mu_{(n)}}$ in $\Gamma$ è dato dalla formula:

$$
\mu(n)=\frac{1}{\lambda} N^{3}(n) \prod_{i}^{1 \ldots h}\left(1-\frac{1}{N^{2}\left(p_{i}\right)}\right)
$$

essendo $N(n)$ la norma del numero $n$, il prodotto esteso a tutti i fattori primi $p_{1}, p_{2}, \ldots, p_{h}$ essenzialmente distinti di $n$, e $\lambda=1$ per $n= \pm 2 \varepsilon^{p},(\rho=0,1,2)$ e $\lambda=2$ in ogni altro caso ( $\$ 1$ ).

Il poliedro fondamentale del gruppo $\Gamma_{\mu(n)}$ ha almeno $\mu(n): 3 N(n)$ vertici impropri, e il gruppo $\bar{\Gamma}_{\mu(n)}^{0}$ ottenuto da $\Gamma_{\mu(n)}$ ampliandolo con la rotazione $z^{\prime}=-z$ e la riflessione $z^{\prime}=z_{0}$ non puó limitarsi per $n$ intero razionale pari, diverso da 2, con un numero finito di piani e sfere di riflessione del gruppo stesso (\$ 2). Le sostituzioni generatrici del gruppo $\bar{\Gamma}$ ottenuto da $\Gamma$ ampliandolo con la rotazione $z^{\prime}=-z$ sono :

$$
S=\left(\begin{array}{ll}
0, & 1 \\
1, & 0
\end{array}\right), \quad T=\left(\begin{array}{cc}
0, & 1 \\
-1, & 1
\end{array}\right), \quad U=\left(\begin{array}{cc}
0, & -\varepsilon \\
\varepsilon^{z}, & 0
\end{array}\right)
$$

caratterizzate dalle relazioni :

$$
S^{2}=1, \quad T^{3}=1, \quad U^{2}=1, \quad(S U)^{6}=1, \quad(T U)^{3}=1, \quad\left(S T^{2}\right)^{2}=1 \quad(\S 3) .
$$

Indicando con $G_{2 \mu_{(n)}}$ il gruppo modulare finito di ordine $2 \mu(n)$ mediante il quale $\bar{\Gamma}$ si rappresenta per $\Gamma_{\mu(n)}$, si trova che nell'ipotesi che il numero

(5) Cfr. la nota dell'A., loc. cit. $\left(^{2}\right)$. 
$n=n_{1}+n_{2} \varepsilon$ sia primo con $2(1-\varepsilon)$, le $s, T, U$ considerate come sostiturioni generatrici di questo gruppo oltre che dalle (2) sono legate dalle relazioni:

$$
\begin{aligned}
& {\left[(U S)^{2}(T U)^{2}\right]^{n_{1}}\left[(S U)^{2}(T U)^{2}(S U)^{2}\right]^{n_{2}}=1,} \\
& {\left[(U S)^{2}(T U)^{2}\right]^{-n_{2}}\left[(S U)^{2}(T U)^{2}(S U)^{2}\right]^{n_{1}-n_{2}}=1,}
\end{aligned}
$$

e ne segue il teorema: Se $i$ legami fra tre operazioni di nalura qual siasi S, T, U sono espressi dalle (2) e (3) (o da conseguenze di queste) ed esse sono atte a generare un gruppo finito, l'ordine del gruppo e $2 \mu(\mathrm{n})$ con $\mu(\mathrm{n})$ espresso dalla (1) (\$ 4 e $\S 5)$.

Nel $\$ 6$ si dimostra che il teorema è vero per i noduli $2^{\lambda}(1-\varepsilon)^{4} m$ con $m$ primo con $2(1-\varepsilon) ; \lambda=0,1 ; \mu=0,1$; e come esempio si studiano i gruppi $G_{2 \mu(1-\varepsilon)}$ e $G_{2 \mu(2)}$ isomorfi rispettivamente coi gruppi ampliati del tetraedro e dell' icosaedro regolare.

Nel \& 7 infine si dimostra che il teorema non sussiste per $i$ moduli $4 m,(1-\varepsilon)^{3} m$ qualunque sia $m$. Tali casi eccezionali sono conseguenza dei due notevoli teoremi:

a) Tutte le sostituzioni unimodulari $\left(\begin{array}{cc}1+4 m a, & 4 m b \\ 4 m c, & 1+4 m d\end{array}\right)$, per le quali il simbolo di JAcoBI dei residui quadratici $\left[\frac{c}{1+4 m a}\right]$ abbia il valore +1 formano gruppo.

b) Tutte le sostituzioni unimodulari $\left(\begin{array}{cc}1+3 m(1-\varepsilon) a, & 3 m\left(1-\varepsilon^{2}\right) b \\ 3 m(1-\varepsilon) c, & 1+3 m(1-\varepsilon) d\end{array}\right)$, per le quali il simbolo relativo ai residui cubici $\left|\frac{c}{1+3 m(1-\varepsilon) a}\right|_{3}$ abbia il valore +1 formano gruppo.

\section{§ 1. L'Indice $\mu(n)$ dei sottogruppi $\Gamma_{\mu(n)}$ del gruppo $\Gamma$ di sostitu- ziont lineart unimodulari con coefficienti interi del campo di Jacobi-Eisenstein $\left(1, \varepsilon=\frac{-1+i \sqrt{3}}{2}\right)$. Il polledro fonda- mentale del sottogruppo $\Gamma_{\mu(1-\xi)}$.}

1. Si consideri il gruppo $\Gamma$ di sostituzioni unimodulari

$$
z^{\prime}=\frac{\alpha z+\beta}{\gamma z+\delta} \quad \alpha \delta-\beta \gamma=1,
$$

a coefficienti interi del corpo $K(\varepsilon), \varepsilon=\frac{-1+i \sqrt{3}}{2}$. Siccome il corpo $K(\varepsilon)$ 
(privo di ideali secondari) si comporta come il corpo di Gauss agli effetti della divisibilita $\left(^{(}\right)$, ripetendo i ragionamenti del $\S 1,(\Lambda)\left({ }^{7}\right)$ concluderemo che il sollogruppo $\Gamma_{\mu(n)}$ di sostiluzioni

$$
z^{\prime}=\frac{\alpha z+\beta-\beta}{\gamma z+\delta}, \quad \alpha \delta-\beta \gamma=1, \quad \alpha-1=\delta-1=\beta \equiv \gamma \equiv 0 \quad(\text { mod. } n),
$$

con $\mathrm{n}$ intero del corpo $\mathrm{k}(\varepsilon)$, e un sottogruppo invariante di indice $\mu(\mathrm{n})$, con

$$
\mu(n)=\frac{1}{\lambda} N^{3}(n) \prod_{i}^{1 \ldots h}\left(1-\frac{1}{N^{2}\left(p_{i}\right)}\right),
$$

essendo $\mathrm{N}(\mathrm{n})$ la norma del numero $\mathrm{n}$, il prodollo esteso a tulli $i$ faltori primi $\mathrm{p}_{1}, \mathrm{p}_{2}, \ldots, \mathrm{p}_{\mathrm{h}}$ distinli di $\mathrm{n}, e \operatorname{con} \lambda=1$ per $\mathrm{n}= \pm 2 \varepsilon^{3},(\rho=0,1,2)$, e $\lambda=2$ negli altri casi.

Si avrà ad esempio

$$
\mu(1-\varepsilon)=\frac{1}{2} 3^{3}\left(1-\frac{1}{3^{2}}\right)=12 ; \mu(2)=4^{3}\left(1-\frac{1}{16}\right)=60 .
$$

2. Costruiamo il poliedro fondamentale del gruppo $\Gamma_{1(1-\varepsilon)}$, verificheremo cosi per via geometrica che si ha $\mu(1-\varepsilon)=12$.

Infatti il gruppo di sostituzioni lineari

$$
z^{\prime}=\frac{\alpha z+-\beta}{\gamma z+\delta}, \quad z^{\prime}=\frac{\alpha z_{0}+\beta}{\gamma z_{0}+\delta}, \quad \alpha \delta-\beta \gamma=1, \quad \beta=\gamma \equiv 0 \quad(\bmod .1-\varepsilon),
$$

con $\alpha, \beta, \gamma, \delta$ interi del corpo $K(\varepsilon)$ ha per poliedro fondamentale il tetraedro regolare $T_{0}$ limitato dai tre piani,

e dalla sfera:

$$
\eta=0, \quad \eta=\xi \sqrt{3}, \quad \xi \sqrt{3}+\eta=\sqrt{3}
$$

$$
\left(\xi-\frac{1}{2}\right)^{2}+\left(\eta-\frac{1}{2 \sqrt{ } 3}\right)^{2}+\zeta^{2}=\frac{1}{3}
$$

essendo $(\xi, \eta, \zeta)$ una terna di assi ortogonali con gli assi $\xi$, $\eta$ nel piano limite del semispazio iperbolico, e il semiasse $\zeta>0$ interno allo stesso semi-

$\left(^{6}\right)$ Cfr. al e8. H. BranchI: Lezioni sulla teoria dei numeri algebriei. (Bologna, N. Znnichelli, 1923), p. 101 e seg.

(7) Cfr. G. Sansonv, loe, eit. (1), p. 276 a seg. 
spazio $\left({ }^{8}\right)$, e il gruppo considerato ha l'indice 24 nel gruppo di sostituzioni :

$$
z^{\prime}=\frac{\alpha z+\beta}{\gamma z+\delta}, \quad z^{\prime}=\frac{\alpha z_{0}+\beta}{\gamma z_{0}+\delta}, \quad \alpha \delta-\beta \gamma= \pm 1 .
$$

Associando a $T_{0}$ il simmetrico rispetto al piano $\eta=0$, considerando cioè

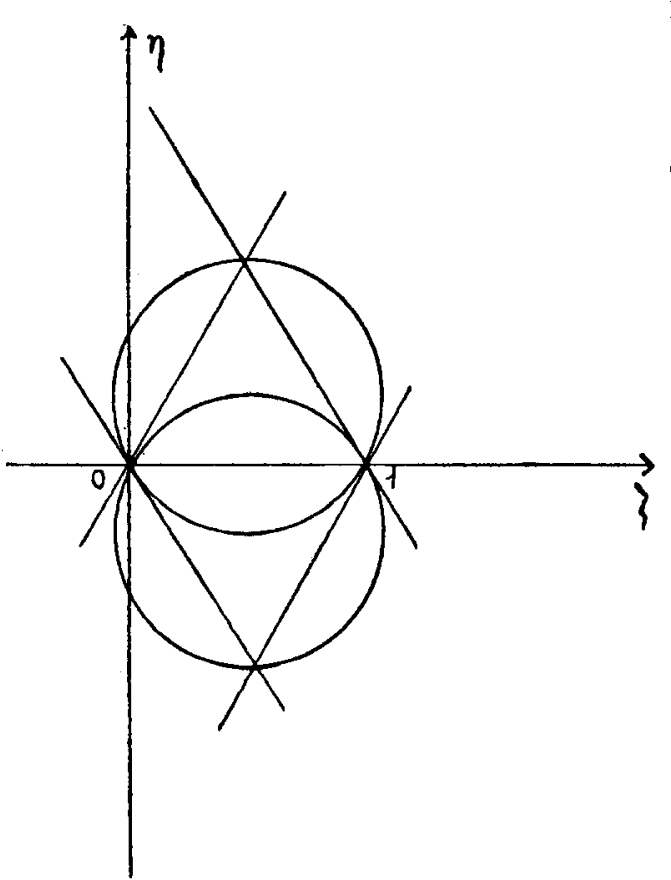

Fig. 1.

il poliedro $T$ limitato dai 4 piani (Fig. 1)

$$
\eta= \pm \xi \vee \overline{3}, \quad \xi \sqrt{3} \pm \eta=\sqrt{3}
$$

e dalle due sfere:

$$
\left(\xi-\frac{1}{2}\right)^{2}+\left(\eta \pm \frac{1}{2 \sqrt{ } 3}\right)^{2}+\zeta^{2}=\frac{1}{3}
$$

esso è il poliedro fondamentale del gruppo

$$
\begin{aligned}
z^{\prime} & =\frac{\alpha z+\beta}{\gamma z+\delta}, \quad(\bmod .1-\varepsilon) . \\
\alpha \delta-\beta \gamma & =1, \quad \beta \equiv \gamma \equiv 0,
\end{aligned}
$$

Tale gruppo coincide col gruppo $\Gamma_{\mu(1-\varepsilon)}$ perchè avendosi

$$
\alpha \delta \equiv 1 \quad(\bmod .1-\varepsilon)
$$

segue

$$
\alpha \equiv \delta \equiv \pm 1 \quad(\bmod .1-\varepsilon)
$$

e cambiando al più il segno ad $\alpha, \beta, \gamma, \delta$, si ha

$$
\alpha \equiv \delta \equiv 1
$$

$(\bmod 1-\varepsilon)$

Segue che il gruppo $\Gamma_{\mu(1-\varepsilon)}$ ha l'indice 24 nel gruppo $\overline{\mathrm{T}}$

$$
z^{\prime}=\frac{\alpha z+\beta}{\gamma z+\delta}, \quad \alpha \delta=\beta \gamma= \pm 1,
$$

e perciò l'indice 12 in $\Gamma$.

(8) Cfr. L. BIANCHI : Sui gruppi di sostituzioni lineavi corrispondenti alle divisioni dello spazio non euclideo in tetraedri e ottaedri regolari. (Rendiconti della $\mathbf{R}$, Accademia dei Lincei, serie V degli Atti, vol. XVIII, $1^{\circ}$ sem. 1909, pp. 645-652), p. 645 e seg. 
Le sostituzioni $Q_{1}, Q_{2}, \ldots, Q_{12}$ che dànuo la rappresentazione di $\Gamma$ per inezzo di $\Gamma_{\mu(1-\xi)}$ formano il gruppo tetraedrale:

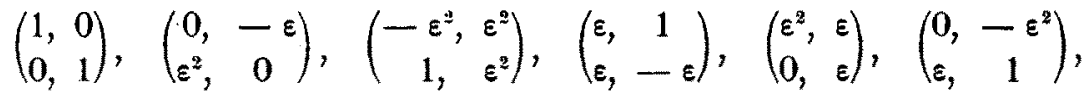

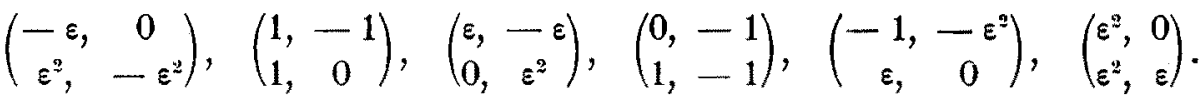

\section{$\S 2$ I campi fondamentali dei gruppl $\Gamma_{\mu(n)}$.}

3. Il tetraedro $T^{\prime}$ limitato dai 3 piani

$$
\xi=\frac{1}{2}, \quad \xi \pm \eta \sqrt{3}=0
$$

e dalla sfera

$$
\xi^{2}+\eta^{2}+\zeta^{2}=1
$$

è il poliedro fondamentale del gruppo $\bar{\Gamma}$ di sostituzioni lineari

$$
z^{\prime}=\frac{\alpha z+\beta}{\gamma z+\delta}, \quad \alpha \delta-\beta \gamma= \pm 1
$$

con $\alpha, \beta, \gamma, \delta$ interi del corpo $K(\varepsilon)\left({ }^{2}\right)$. Associando a $T^{\prime}$ il suo simmetrico rispetto al piano $\xi=\frac{1}{2}$ si ha che la piramide $P$ limitata dai 4 piani

$$
\xi \pm \eta \sqrt{3}=0, \quad \xi \pm \eta \sqrt{3}=1,
$$

e dalle due sfere

$$
\xi^{2}+\eta^{2}+\zeta^{2}=1, \quad(\xi-1)^{2}+\eta^{2}+\zeta^{2}=1
$$

è il poliedro fondamentale del gruppo $\Gamma$ (Fig. 2).

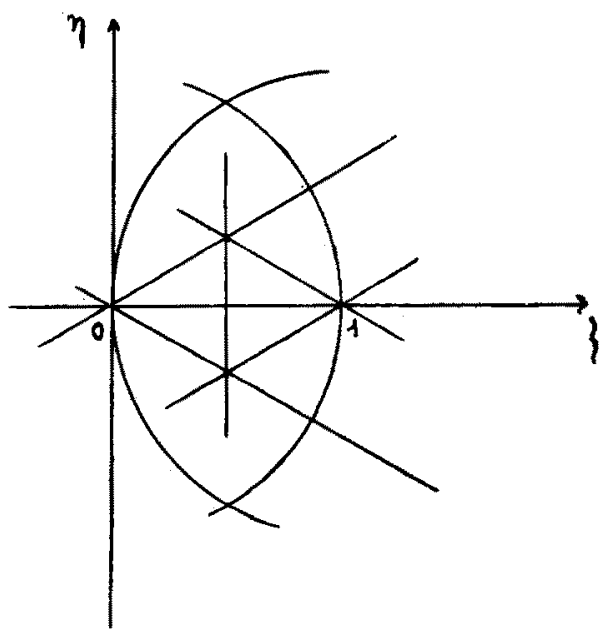

Fig. 2.

Chiamando con $\Sigma$ la divisione regolare del semispazio iperbolico operata dalla piramide $P$ e considerando la rete dei prismi a base quadrangolare determinata dai due sistemi di piani $\xi \pm \eta \sqrt{3}=l$ con $l$ intero arbitrario, troviamo che ad ogni prisma della rete appartiene una cella di $\Sigma$ avente per

$\left({ }^{9}\right)$ Cfr. L. Bianoh, loc. cit. $\left({ }^{3}\right)$, p. 365 . 
vertice il punto all'infinito $V_{\infty}$ del semispazio iperbolico. Escluso il caso $n= \pm(1-\varepsilon)^{p}, \rho=0,1,2$, da noi esaminato al $\$ 1$, i movimenti di $\Gamma_{\mu_{(n)}}$ che lasciano fisso $V_{\infty}$ sono le traslazioni $z^{\prime}=z+n b$ con $b$ intero arbitrario. Segue che nel parallelogramma $Q^{\prime}$ di vertici $0, n, n \varepsilon$, - $n \varepsilon^{2}$ non vi sono punti equivalenti per una traslazione $z^{\prime}=z+n b$ (Fig: 3).

Infatti le traslazioni $z^{\prime}=z+n \varepsilon^{p}, \rho=0,1,2$, inducono in un punto una traslazione equipollente al vettore $\pm n \varepsilon^{p}$ e portano pertanto un punto interno a $Q^{\prime}$ in un punto esterno; le traslazioni $z^{\prime}=z \pm n(1-\varepsilon) \varepsilon^{?}, \rho=0,1,2$ indu-

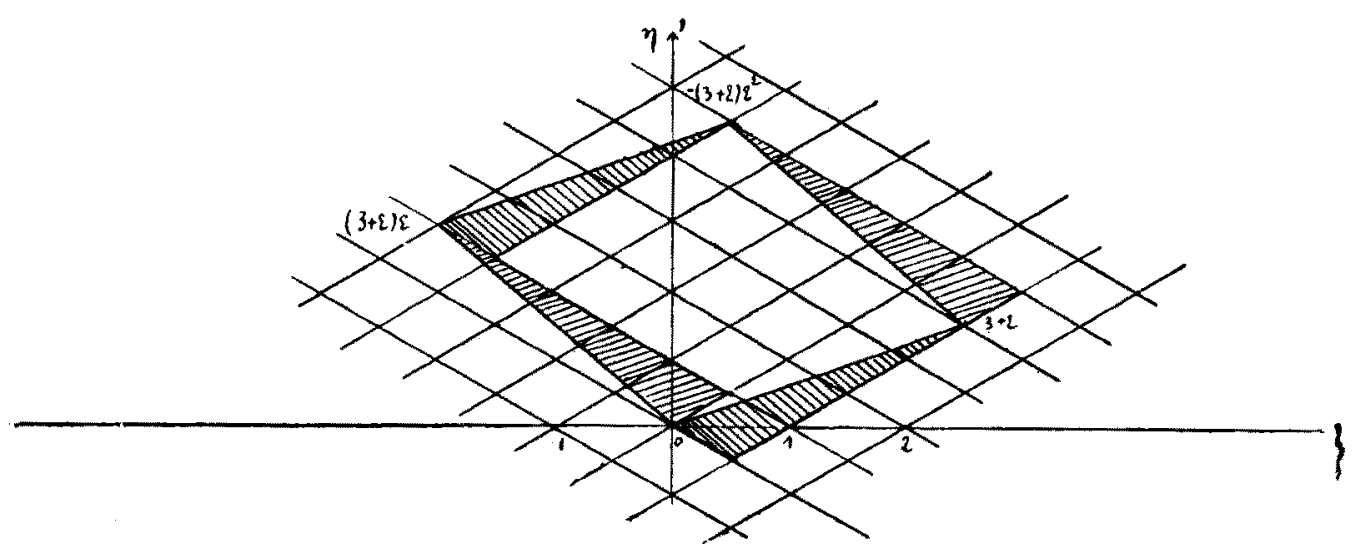

Fig. 3 .

cono in un punto una traslazione di ampiezza uguale alla diagonale di $Q^{\prime}$ che unisce i vertici $n, n \varepsilon$ e per esse i punti interni a $Q^{\prime}$ sono portati in punti esterni ; ogni altra traslazione $z^{\prime}=z+n b$ è di ampiezza maggiore della diagonale maggiore di $Q^{\prime}$, quindi in $Q^{\prime}$ non vi sono punti equivalenti per le traslazioni considerate.

Consideriamo ora il prisma formato dai 4 piani normali al piano limite che segnano su di esso il parallelogrammo $Q^{\prime}$; i puntí interni a questo prisma non sono equivalenti per le sostituzioni di $\Gamma$ che lasciano fisso $V_{\infty}$. Con cambiamenti leciti, a questo prisma possiamo sostituire un insieme $I$ di celle di $\Sigma$ che non sono equivalenti rispetto a $\Gamma_{\mu_{(n)}}$ e tali che ogni altra cella di $\Sigma$ di vertice $V_{\infty}$ sia equivalente ad una cella di $I$ per una traslazione di $\Gamma_{\mu_{(n)}}$.

Le celle di $I$ sono in numero uguale al rapporto tra l' area del parallelogrammo $Q^{\prime}$ che è $N(n) \frac{\sqrt{3}}{2}$ e l'area del parallelogrammo limitato dai 4 piani $\xi \pm \eta \vee 3=0, \xi \pm \eta \sqrt{3}=1$, che è $\frac{1}{6} \sqrt{3}$, cioè sono $3\left(n_{1}^{2}+n_{2}^{2}-n_{1} n_{2}\right)$, (v. Fig. 3 , caso $n=3+\varepsilon)$. 
Ne segue allora $\left({ }^{(0)}\right)$ che si può costruire un poliedro fondamentale $P_{\mu(n)}$ di $\Gamma_{\mu_{(n)}}$ composto di $\mu(n)$ celle congruenti a $P$ tale che ogni cella ne abbia aderente un'altra almeno per un vertice, e che attorno ad ogni suo vertice improprio vi siano $3 N(n)$ celle di $\Sigma$ e quindi con $\mu(n): 3 N(n)$ vertici improprii, e perciò questo numero è il minor numero di vertici impropri del poliedro fondamentale di $\Gamma_{\mu_{(n)}}$.

4. $\dot{\mathrm{E}}$ ancora facile provare che supposto il modulo $n$ intero razionale pari, e diverso da 2, ampliando il gruppo $\Gamma_{\mu(n)}$ con la rotazione $z^{\prime}=-z$ e la riflessione $z^{\prime}=z_{0}$ si ha un gruppo $\overline{\mathbf{\Gamma}}_{\mu(n)}$ di cui il poliedro fondamentale non può limitarsi con un numero finito di facce e sfere di riflessione del gruppo stesso. Infatti la riflessione di $\bar{\Gamma}_{\mu(n)}$ sono:

$$
z^{\prime}=\frac{\left(1+n a_{1}+n a_{2} \varepsilon\right) z_{0}+n i b_{1} \sqrt{3}}{n i c_{1} \sqrt{3} z_{0}+\left(1+n a_{1}+n a_{2} \varepsilon^{2}\right)}, \quad z^{\prime}=\frac{\left(1+n a_{1}+n a_{2} \varepsilon\right) z_{0}+n b_{1}}{n c_{1} z_{0}-\left(1+n a_{1}+n a_{2} \varepsilon^{2}\right)},
$$

essendo rispettivamente

$$
\begin{aligned}
& \left(1+n a_{1}+n a_{2}\right)^{2}-3 n a_{2}\left(1+n a_{1}\right)+3 n^{2} b_{1} c_{1}=1, \\
& \left(1+n a_{1}+n a_{2}\right)^{2}-3 n a_{2}\left(1+n a_{1}\right)+n^{2} b_{1} c_{1}=1,
\end{aligned}
$$

e per $c_{1} \neq 0$ le corrispondenti sfere di riflessione hanno per equazione:

$$
\begin{aligned}
& \left(\xi-\frac{a_{2}}{2 c_{1}}\right)^{2}+\left(\eta+\frac{\left.2\left(1+n a_{1}\right)-n a_{2}\right)}{2 n c_{1} \sqrt{3}}\right)^{2}+\zeta^{2}=\frac{1}{3 n^{2} c_{1}^{2}}, \\
& \left(\xi-\frac{2\left(1+n a_{1}\right)-n a_{2}}{2 n c_{1}}\right)^{2}+\left(\eta-\frac{a_{2} \sqrt{3}}{2 c_{1}}\right)^{2}+\zeta^{2}=\frac{1}{n^{2} c_{1}^{2}},
\end{aligned}
$$

mentre per $c_{1}=0$, avendo supposto $n \neq 2$, si ha $a_{2}=a_{1}=0$ e i corrispondenti piani di riflessione hanno per equazione $\xi=\frac{n}{2} b, \eta=\frac{n}{2} b \sqrt{3}$, con $b$ intero qualunque.

Proviamo che il punto di coordinate $\left(\frac{q}{n}, \frac{q \sqrt{3}}{n}, 0\right)$, con $q$ primo con $n$ non solo non è sull' intersezione di due piani di riflessione (evidente) ma non è nè interno nè su qualsiasi sfera di riflessione.

(10) Cfr. p. 287 (A). 
Infatti $\left(\frac{q}{n}, \frac{q \sqrt{3}}{n}, 0\right)$ può essere interno o su una sfera di riflessione quando si abbia :

$$
\begin{aligned}
& {\left[\frac{q}{n}-\frac{a_{2}}{2 c_{1}}\right]^{2}+\left[\frac{q \sqrt{3}}{n}+\frac{2\left(1+n a_{1}\right)-n a_{2}}{2 n c_{1} \sqrt{3}}\right]^{2} \leq \frac{1}{3 n^{2} c_{1}^{2}},} \\
& {\left[\frac{q}{n}-\frac{2\left(1+n a_{1}\right)-n a_{2}}{2 n c_{1}}\right]^{2}+\left[\frac{q \sqrt{3}}{n}-\frac{a_{2} \sqrt{3}}{2 c_{1}}\right]^{2} \leq \frac{1}{n^{2} c_{1}^{2}},}
\end{aligned}
$$

ovvero

$$
\begin{aligned}
& 3\left(2 c_{1} q-n a_{2}\right)^{2}+\left[6 q c_{1}+2\left(1+n a_{1}\right)-n a_{2}\right]^{2} \leq 4 \\
& {\left[2 c_{1} q-2\left(1+n a_{1}\right)+n a_{2}\right]^{2}+3\left(2 c_{1} q-n a_{2}\right)^{2} \leq 4}
\end{aligned}
$$

e per la parità di $n$, deve essere rispettivamente:

$$
\begin{aligned}
& 2 c_{1} q-\dot{n} a_{2}=0, \quad 6 c_{1} q+2\left(1+n a_{1}\right)-n a_{2}=0 \\
& 2 c_{1} q-2\left(1+n a_{1}\right)+n a_{2}=0, \quad 2 c_{1} q=n a_{2}
\end{aligned}
$$

dalle quali $-2 c_{1} q=1+n a_{1} ; \quad 2 c_{1} q=1+n a_{1}$ le quali sono impossibili avendo supposto $n$ pari.

$\mathrm{Ne}$ segue, come si era prima affermato, che il poliedro fondamentale di $\overline{\mathbf{\Gamma}}_{\mu(n)}$ non puó limitarsi con un numero finito di piani e sfere di riflessioni del gruppo.

\section{§ 3. Relazioni fondamentali fra le sostituzioni generatrici del gruppo $\bar{\Gamma}$.}

5. Nella Memoria citata del BranchI ( $\left.{ }^{14}\right)$ è dimostrato che il gruppo $\widetilde{\Gamma}^{0}$ di sostituzioni di prima e seconda specie

$$
z^{\prime}=\frac{\alpha z+\beta}{\gamma z+\delta}, \quad z^{\prime}=\frac{\alpha z_{0}+\beta}{\gamma z_{0}+\delta}, \quad \alpha \delta-\beta \gamma= \pm 1
$$

$\alpha_{;} \beta, \gamma, \delta$, interi del corpo $K(\varepsilon)$ ha per poliedro fondamentale il tetraedro $T$ con un vertice improprio limitato dalle 4 facce:

$$
\eta=0, \quad \xi=\frac{1}{2}, \quad \xi=\eta \sqrt{3}, \quad \xi^{2}+\eta^{2}+\xi^{2}=1
$$

(i1) L. Bianchi, loc. cit. $\left({ }^{3}\right)$, p. 365 . 
Il gruppo $\bar{\Gamma}^{0}$ si può quindi generare con le 4 riflessioni :

$$
z^{\prime}=z_{0}, \quad z^{\prime}=-z_{0}+1, \quad z^{\prime}=\frac{\varepsilon z_{0}}{-\varepsilon^{2}}, \quad z^{\prime}=\frac{1}{z_{0}},
$$

che indicheremo rispettivamente con $A, B, C, D$, si ha perciò

$$
A=\left(\begin{array}{ll}
1, & 0 \\
0, & 1
\end{array}\right) z_{0}, \quad B=\left(\begin{array}{cc}
-1, & 1 \\
0, & 1
\end{array}\right) z_{0}, \quad C=\left(\begin{array}{cc}
\varepsilon, & 0 \\
0, & -\varepsilon^{2}
\end{array}\right) z_{0}, \quad D=\left(\begin{array}{ll}
0, & 1 \\
1, & 0
\end{array}\right) z_{0},
$$

con

$$
A^{2}=1, \quad B^{2}=1, \quad C^{2}=1, \quad D^{2}=1
$$

Avendosi poi

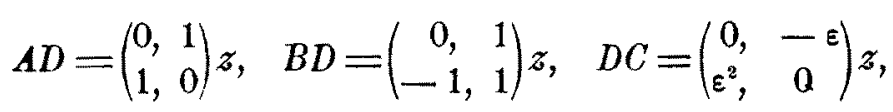

quindi

$$
(A D)^{2}=1, \quad(B D)^{3}=1, \quad(C D)^{2}=1, \quad D A=A D, \quad D B=(B D)^{2}, \quad D C=C D
$$

segue che le sostituzioni del gruppo $\bar{\Gamma}$

$$
z^{\prime}=\frac{\alpha z+\beta}{\gamma z+\delta}, \quad \alpha \delta-\beta \gamma= \pm 1,
$$

$(\alpha, \beta, \delta, \gamma$ interi del corpo $K(\varepsilon))$ si generano con le 3 sostiluzioni ellittiche:

$$
S=A D=\left(\begin{array}{ll}
0, & 1 \\
1, & 0
\end{array}\right), \quad T=B D=\left(\begin{array}{cc}
0, & 1 \\
-1, & 1
\end{array}\right), \quad U=C D=\left(\begin{array}{cc}
1, & -\varepsilon \\
\varepsilon^{2}, & 0
\end{array}\right)
$$

con

$$
S^{2}=1, \quad T^{3}=1, \quad U^{2}=1 .
$$

Vogliamo trovare tutte le relazioni indipendenti tra le $S, T, U$. Si consideri il tetraediro $T^{\prime}$ ottenuto assaciando a $T$ il simmetrico rispetto al piano $\eta=0$ cioè il tetraedro limitato dai 4 piani (Fig. 4):

$$
\xi= \pm \eta \sqrt{3}, \quad \xi=\frac{1}{2}, \quad \xi^{2}+\eta^{2}+\zeta^{2}=1
$$

i cui vertici sono:

$$
V_{0}=(0,0,1), \quad V_{1}=\left(\frac{1}{2}, \frac{1}{2 \sqrt{3}}, \sqrt{\frac{2}{3}}\right), \quad V_{2}=\left(-\frac{1}{2}, \frac{1}{2 \sqrt{3}}, \sqrt{\frac{2}{3}}\right), \quad V_{3}=\infty
$$




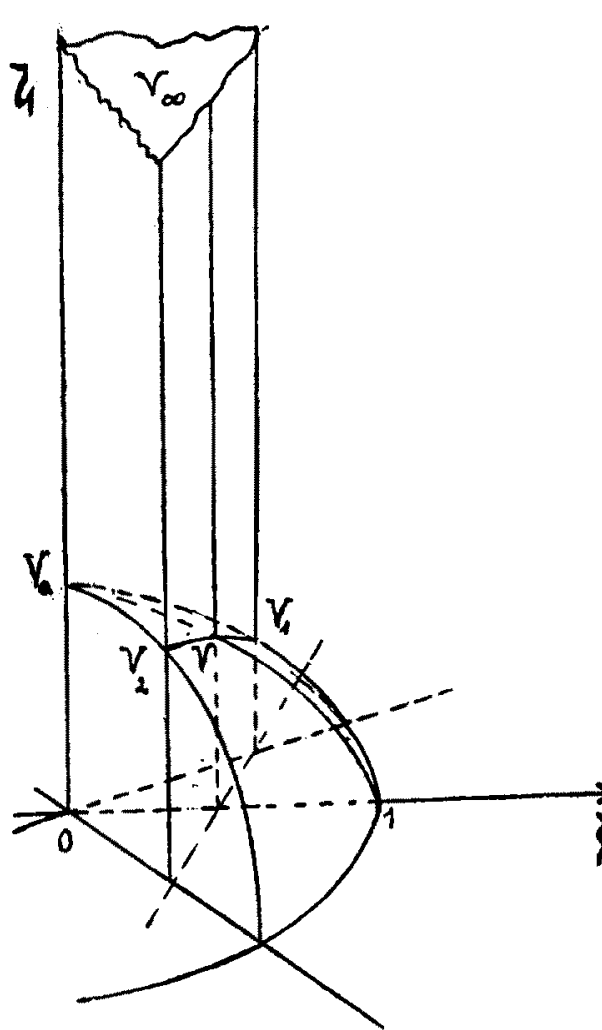

Fig. 4. ed indichi $V=\left(\frac{1}{2}, 0, \frac{\sqrt{3}}{2}\right)$ il punto medio dell' arco $\widehat{V}_{1}$. E facile provare ("2) che ogni relazione fra le sostituzioni del gruppo $\bar{\Gamma}$ è un prodotto di un numero finito di trasformate delle rotazioni attorno agli spigoli

$$
\begin{aligned}
& V_{0} V ; \quad V_{1} V_{2} ; \quad V_{0} V_{1} ; \quad V_{0} V_{2} ; \\
& V_{0} V_{\infty} ; \quad V_{1} V_{\infty} ; \quad V_{2} V_{\infty} ; \quad V V_{\infty} ;
\end{aligned}
$$

le quali hanno rispettivamente per espressione :

$$
S^{2}, \quad T^{3}, \quad U^{2}, \quad S\left(U^{*}\right) S, \quad(S U)^{6},
$$

$(T U)^{3}, \quad S T^{2}(T U)^{3} T S, \quad\left(S T^{2}\right)^{2}$,

e di queste la quarta è conseguenza della terza, la settima della sesta, restano fra le $S, T, U$, le relazioni indipendenti :

$$
\begin{gathered}
S^{2}=1, \quad T^{3}=1, \quad U^{2}=1, \\
(S U)^{6}=1, \quad(T U)^{3}=1, \quad\left(S T^{2}\right)^{2}=1 .
\end{gathered}
$$

\section{\$ 4. Sulla Indipendenza delle sostituzioni $s, T, U$ considerate come sostituzioni generatricl del gruppo finito $G_{2 \mu(n)}$, e sulle loro relazioni caratteristiche nel gruppo stesso.}

6. Il gruppo $\Gamma_{\mu(n)}$ ha nel gruppo $\bar{\Gamma}$ l'indice $2 \mu(n)$, e le $2 \mu(n)$ sostituzioni a determinante \pm 1 con le quali $\bar{\Gamma}$ si rappresenta per $\Gamma_{\mu(n)}$ formano un gruppo finito $G_{\Omega_{(n)}}$ di ordine $2 \mu(n)$, ove si considerino percio come identiche due sostituzioni i cui elementi siano ordinatamente congrui (modulo $n$ ), oppure con gli elementi di una congrua ordinatamente agli elementi opposti dell'altra (modulo $n$ ). Se costruiamo i tre gruppi finiti di ordine 6, 12, 12 generati dalle operazioni $T, S ; S, U ; U, T$ si verifica facilmente che qualunque sia il modulo $n$, non puó una qualunque delle sostituzioni $U, T, S$ esprimersi come un

(12) Cfr. pag. 295 (A). 
prodotto formato con le altre due sostituzioni, ossia le $S, T, U$ considerate come operazioni del gruppo $G_{2,4(n)}$ sono indipendenti.

Per determinare delle altre relazioni fra le $S, T, U$ oltre le (I), troviamo le sostituzioni di $\Gamma_{\mu(n)}$ che lasciano fisso $V_{\infty}$. Escluso il caso $n= \pm(1-\varepsilon) \varepsilon^{p}$, $\rho=0,1,2$ esse hanno la forma:

$$
z^{\prime}=\left(\begin{array}{cc}
1, & n l \\
0, & 1
\end{array}\right) z
$$

e posto

quindi

$$
n=n_{1}+n_{2} \varepsilon, \quad l=l_{1}+l_{2} \varepsilon
$$

$$
\left(n_{1}+n_{2} \varepsilon\right)\left(l_{1}+l_{2} \varepsilon\right)=l_{1}\left(n_{1}+n_{2} \varepsilon\right)+l_{2}\left[-n_{2}+\left(n_{1}-n_{2}\right) \varepsilon\right]
$$

le sostituzioni precedenti si compongono con le potenze delle traslazioni

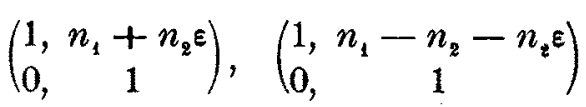

e per queste nel gruppo $G_{2 \mu_{(n)}}$ si ha:

$$
\left(\begin{array}{cc}
1, & n_{1}+n_{2} \varepsilon \\
0, & 1
\end{array}\right)=1, \quad\left(\begin{array}{l}
1, n_{1}-n_{2}-n_{\mathrm{z}} \varepsilon \\
0,
\end{array}\right)=1
$$

Ma si ha:

$$
\left(\begin{array}{ll}
1, & 1 \\
0, & 1
\end{array}\right)=(U S)^{2}(T U)^{2}, \quad\left(\begin{array}{ll}
1, & \varepsilon \\
0, & 1
\end{array}\right)=(S U)^{2}(T U)^{2}(S U)^{2},
$$

e le precedenti diventano:

$$
\begin{aligned}
& {\left[(U S)^{2}(T U)^{2}\right]^{n_{1}}\left[(S U)^{2}(T U)^{2}(S U)^{2}\right]^{n_{2}}=1,} \\
& {\left[(U S)^{2}(T U)^{2}\right]^{-n_{3}}\left[(S U)^{2}(T U)^{2}(S U)^{2}\right]^{n_{1}-n_{2}}=1 .}
\end{aligned}
$$

Inversamente dalle (II) e (I) segue che ogni sostituzione $z^{\prime}=\left(\begin{array}{cc}1, & n l \\ 0, & 1\end{array}\right) z$ nel gruppo $G_{2 \mu(n)}$ si rappresenta con l'identita. Basterà provare per questo che le due sostituzioni $(U S)^{2}(T U)^{2}$ e $(S U)^{2}(T U)^{2}(S U)^{2}$ sono permutabili, cioè basterà provare che:

$$
(U S)^{2}(T U)^{2}(S U)^{2}(T U)^{2}(S U)^{2}=(S U)^{2}(T U)^{2}(S U)^{2}(U S)^{2}(T U)^{2}
$$

ovvero avendosi $(S U)^{2}(U S)^{2}=1$ e $(T U)^{3}=1$, basterà provare che :

$$
(U S)^{2}(T U)^{9}(S U)^{2}(T U)^{2}(S U)^{2}=(S U)^{2} T U
$$

e moltiplicando a sinistra per $(S U)^{2}$ e a destra per $(T U)^{2}(S U)^{2}$ occorre verifi- 
care che :

$$
(T U)^{2}(S U)^{2}(T U)^{2}(S U)^{9}(T U)^{2}(S U)^{2}=\left[(T U)^{2}(S U)^{2}\right]^{3}=1
$$

Ma è

$$
(T)^{2}(S U)^{2}=U T^{2}(S U)^{2}=U S T U S U=U S(T U)(U S)^{-1}
$$

perciò

$$
\left[(T U)^{9}(S U)^{2}\right]^{3}=\left[(U S)(T U)(U S)^{-1}\right]^{3}=(U S)(T U)^{3}(U S)^{-1}=1 .
$$

Notiamo infine che ogni sostituzione parabolica del gruppo $\Gamma_{\mu_{(n)}}$ è una trasformata della (4) $\left({ }^{13}\right)$, segue che le (II) esprimono le relazioni fra le $S, T, U$ che si hanno dalla condizione che le sostituzioni paraboliche del gruppo $\Gamma_{\mu(n)}$ si rappresentano nel gruppo con l'unita. Dimostreremo nel $\$$ seguente che le (I) e (II) rappresentano tutte le relazioni indipendenti tra le operazioni del gruppo $G_{\mathrm{g} \mu(n)}$ quando il modulo $n$ non è divisibile per $2^{9}$, od $(1-\varepsilon)^{3}$ cioè ogni relazione fra le $S, T, U$ in $G_{\mu(n)}$ è conseguenza delle (I) e (II), segue quindi il teorema: Se $i$ legami fra tre operazioni S, T, U sono espressi dalle relazioni:

$(\mathrm{I})^{*} \quad S^{2}=1, \quad T^{3}=1, \quad U^{2}=1, \quad(S U)^{6}=1, \quad(T U)^{3}=1, \quad\left(S T^{2}\right)^{2}=1$,

$$
\begin{aligned}
& {\left[(U S)^{2}(T U)^{2}\right]^{n_{1}}\left[(S U)^{2}(T U)^{2}(S U)^{2}\right]^{n_{2}}=1,} \\
& {\left[\left.(U S)^{2}(T U)^{2}\right|^{-n_{2}}\left[(S U)^{2}(T U)^{2}(S U)^{2}\right]^{n_{1}-n_{z}}=1,\right.}
\end{aligned}
$$

(o da loro conseguenze) e il numero $\mathrm{n}=\mathrm{n}_{1}+\mathrm{n}_{2} \varepsilon$ non $\dot{e}$ divisibile per $2^{2}$ $e(1-\varepsilon)^{3}$, ed esse sono atte a generare un gruppo finito, il gruppo da esse generato è isomorfo oloedricamente col gruppo modulare $\mathrm{G}_{2 \mu_{(n)}}$ di ordine

$$
2 \mu(n)=\frac{2}{\lambda} N^{3}(n) \prod_{i}^{1 \ldots h}\left(1-\frac{1}{N^{2}\left(p_{i}\right)}\right)
$$

prendendo $\lambda=1$ per $\mathrm{n}= \pm 2 \varepsilon^{\circ},(\rho=0,1,2)$ e $\lambda=2$ negli altri casi, e il prodolto esteso a tutti $i$ fattori primi essenzialmente distinti di $n_{1}+n_{z} \varepsilon$.

\section{\$ 5. Dimostrazione del teorema fondamentale sui gruppi $G_{2 \mu(n)}$ nel caso di moduli primi con $2(1-\varepsilon)$.}

7. Unil sostituzione $\Omega=S^{\alpha_{1}} T^{\beta_{1}} U^{\gamma_{1}} S^{\alpha_{a}} T^{\beta_{9}} U^{\gamma_{9}} \ldots$ rappresenta nel gruppo $G_{\text {qu(n) }}$ l'identità quando si abbia $\Omega=1$, oppure

$$
\Omega=\left(\begin{array}{ll}
1, & 0 \\
0, & 1
\end{array}\right) \quad(\bmod . n) .
$$

(13) Cfr. p. 304 (A). 
Nel primo caso, come si è detto al $\$ 3$ la $\mathbf{\Omega}$ è conseguenza delle (1), nel secondo caso, $Q$ ha la forma:

$$
\mathbf{Q}=\left(\begin{array}{cc}
1+n a, & n b \\
n c, & 1+n d
\end{array}\right)
$$

e il teorema sarà dimostrato ove si provi che per $n$ non divisibile per $2^{z}$, $(1-\varepsilon)^{3}$ la $(6)$ è il prodotto di un numero finito di trasformate della sostituzione parabolica $\left(\begin{array}{cc}1, n k \\ 0, & 1\end{array}\right)$. Seguiremo per questo la via già tenuta nella memoria citata ${ }^{14}$ ) riconducendo il teorema a quello del Dick, (gruppo modulare con coefficienti del campo minimo di razionalità).

Premettiamo le seguenti osservazioni $\left({ }^{15}\right)$ :

Osservazione $1^{\mathrm{a}}$. Se la sostituzione unimodulare $\left(\begin{array}{cc}1+\mathrm{na}, & \mathrm{nb} \\ \mathrm{nc}, & 1+\mathrm{nd}\end{array}\right)$ è un prodotto di sostituzioni paraboliche di $\mathrm{\Gamma}_{\mu(\mathrm{n})}$, anche la sostituzione unimodulare $\left(\begin{array}{cc}1+\mathrm{na}, & \mathrm{nb}^{\prime} \\ \mathrm{nc}, & 1+\mathrm{nd}^{\prime}\end{array}\right)$ e un prodotto di sostituzioni paraboliche di $\Gamma_{\mu(\mathbf{n})}$.

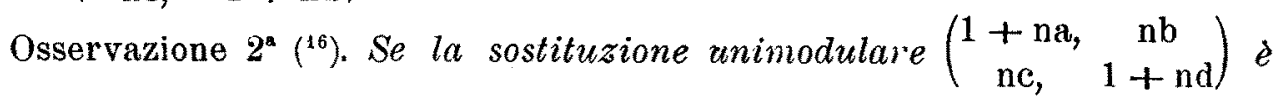
un prodotto di sostituzioni paraboliche di $\Gamma_{\mu_{(\mathrm{n})}}$ anche la sostituzione $\left(\begin{array}{l}1+\mathrm{na}, \pm \mathrm{nb \varepsilon ^{-p }} \\ \pm \mathrm{nc \varepsilon ^{p }}, 1+\mathrm{nd}\end{array}\right)$ è un prodotto di sostituzioni paraboliche di $\Gamma_{1(n)}$.

Seguono i soliti Lemmi, ci limiteremo però a dare quelle dimostrazioni che subiscono qualche modificazione.

Lemma $1^{\circ}$. Data una sostituzione $\Omega=\left(\begin{array}{cc}1+\mathrm{na}, & \mathrm{nb} \\ \mathrm{nc}, & 1+\mathrm{nd}\end{array}\right)$ esiste una sua trasformata, che moltiplicata per sostituzioni paraboliche di $\Gamma_{\mu(\mathrm{n})}$ assume la forma $\left(\begin{array}{cc}1+\mathrm{N} \alpha, & \mathrm{N} \beta \\ \mathrm{N} \gamma, & 1+\mathrm{N} \delta\end{array}\right)$ con $\mathrm{N}$ multiplo arbitrario di $\mathrm{n}$.

Sia $N=n q$, e supponiamo dapprima che nella $\Omega$ sia $c$ primo con $q$. Sia allora $x$ radice della congruenza $a-c x \equiv 0$ (mod. $q$ ), la trasformata di $\mathbf{Q}$ :

$$
\Omega_{1}=\left(\begin{array}{ll}
1, x \\
0, & 1
\end{array}\right) \Omega\left(\begin{array}{cc}
1,-x \\
0, & 1
\end{array}\right)=\left(\begin{array}{cc}
1+n(a-c x), & n b_{1} \\
n c, & 1+n d_{1}
\end{array}\right)=\left(\begin{array}{cc}
1+n q a_{1}, & n b_{1} \\
n c, & 1+n d_{1}
\end{array}\right)
$$

ha già il primo coefficiente $\equiv 1$ modulo $n q=N$.

(14) Cfr. 10 , p. 311 e seg. (A).

$\left.{ }^{15}\right)$ Cfr. loc. eit. $\left({ }^{2}\right)$, n. 2.

${ }^{(6)}$ Cfr. loc. eit. $\left({ }^{2}\right)$, n. 2. 
Sia ora $\underset{r}{c \mid \frac{q}{k}}, b_{1} \frac{\mid q}{l}$ cioè $c=k q+r, b_{1}=l q+s$ si ha:

$$
\Omega_{2}=\Omega_{1}\left(\begin{array}{cc}
1, & 0 \\
-n r, & 1
\end{array}\right)\left(\begin{array}{cc}
1, & -n s \\
0, & 1
\end{array}\right)=\left(\begin{array}{cc}
1+n q \alpha, & n q \beta \\
n q \gamma, & 1+n d_{2}
\end{array}\right)
$$

ed essendo $\Omega_{2}$ unimodulare, è $d_{2} \equiv 0($ mod. $q)$ cioè $d_{2}=q \delta$ e quindi la $\Omega_{2}$ ha la forma richiesta.

Quando nella $\mathbf{Q}$ non sia $c$ primo con $q$, se poniamo $y$ uguale al prodotto di tutti i fattori primi di $q$ non contenuti in $c$, nella sostituzione:

$$
\mathbf{\Omega}_{3}=\mathbf{\Omega}\left(\begin{array}{cc}
1, & 0 \\
n y, & 1
\end{array}\right)=\left(\begin{array}{cc}
1+n a, & n b \\
1+n[c+y(1+n a)], & 1+n d_{1}
\end{array}\right)
$$

è $c+y(1+n a)$ primo con $q$ e perciò possiamo operare sulla $\Omega_{3}$ come si è operato prima sulla $\Omega$.

Più in particolare ragionando come nel Lemma $1^{\circ}$ del $\& 10$ della Memoria citata (A) si ha:

Lemma $2^{\circ}$. Data una sostituzione unimodulare (6), si puo moltiplicarla a destra e a sinistra per opportune trasformate della sostituzione parabolica $\left(\begin{array}{c}1, \mathrm{nk} \\ 0,1\end{array}\right)$ in guisa che la sostituzione prodotta abbia la forma:

$$
\mathbf{\Omega}^{\prime}=\left(\begin{array}{cc}
1+N^{2} \alpha, & N \beta \\
N_{\gamma} & 1+N^{2} \delta
\end{array}\right)
$$

con $\alpha, \beta, \gamma, \delta$ interi del corpo $\mathrm{K}(\varepsilon), \mathrm{N}$ intero razionale, ( $\mathrm{N}$ multiplo arbitrario non nullo di $\mathrm{n}$ ) e quando ci occorra $\mathrm{N} \equiv 0$ (mod. 12).

Nel seguito $N$, ove non si avverta espressamente indica un numero intero razionale.

Lemma $3^{\circ}$. Se una sostituzione $\Omega$ ha la forma (7), è lecito moltiplicarla per una sostituzione parabolica di $\Gamma_{\mu_{(n)}}$ in guisa che la parte reale $e$ il coefficiente di $\varepsilon$ di $\gamma$ siano primi tra loro.

Si ha infatti :

$$
\left(\begin{array}{cc}
1, & 0 \\
N x, & 1
\end{array}\right) \mathbf{\Omega}=\left(\begin{array}{cc}
1+N^{2} \alpha, & N \beta \\
N\left[\gamma+x\left(1+N^{2} \delta\right)\right], & 1+N^{2} \delta
\end{array}\right)=\left(\begin{array}{cc}
1+N^{2} \alpha, & N \beta \\
N \pi, & 1+N^{2} \delta
\end{array}\right)
$$

con $\pi=\gamma+x\left(1+N^{2} \delta\right)$. Supposto $1+N^{2} \delta$ primo con 6 , si puó trovare un $x_{0}$ tale che sia

$$
\gamma+x_{0}\left(1+N^{2} \delta\right) \equiv 2-\varepsilon \quad(\bmod .6)
$$


e posto $x=x_{0}+6 t$ si ha allora :

$$
\pi=\gamma+x_{0}\left(1+N^{2} \tilde{\delta}\right)+6\left(1+N^{2} \delta\right) t
$$

Il numero $6\left(1+N^{2} \delta\right)$ è per le ipotesi fatte primo con $\gamma+x_{0}\left(1+N^{2} \delta\right)$, si può perció assegnare all'indeterminata $t\left({ }^{17}\right)$ un valore per il quale $\pi$ risulti primo complesso; esso è per la (8) di primo grado, e perció ha la parte reale e il coefficiente di $\varepsilon$ primi tra loro. Quando non sia $1-N^{2} \delta$ primo con 6 , posto $h$ uguale al prodotto dei fattori primi di 6 non contenuti in $1+N^{2} 5$, la sostituzione

$$
\mathbf{\Omega}_{1}=\mathbf{\Omega}\left(\begin{array}{cc}
1, & 0 \\
N h, & 1
\end{array}\right)=\left(\begin{array}{cc}
1+N^{2} \alpha, & N \beta \\
N \gamma_{1}, & 1+N^{2} \delta_{1}
\end{array}\right)
$$

ha il quarto coefficiente primo con 6.

Lemma $4^{\circ}$. Se nella sostituzione $\Omega=\left(\begin{array}{cc}1+\mathrm{N}^{2}\left(\mathrm{a}_{1}+\mathrm{a}_{2} \varepsilon\right), & \mathrm{N}\left(\mathrm{b}_{1}+\mathrm{b}_{2} \varepsilon\right) \\ \mathrm{N}\left(\mathrm{c}_{1}+\mathrm{c}_{2} \varepsilon\right), & 1+\mathrm{N}^{2}\left(\mathrm{~d}_{1}+\mathrm{d}_{2} \varepsilon\right)\end{array}\right)$, $\mathrm{c}_{1}$ e $\mathrm{c}_{2}$ sono primi tra loro, ad essa, salvo moltiplicarla per sostituzioni paraboliche di $\Gamma_{1(\mathrm{n})}$ si pud dare la forma:

$$
\left(\begin{array}{cc}
1+N^{2} \alpha, & N\left(c_{1}+c_{2} \varepsilon^{2}\right) \beta \\
N\left(c_{1}+c_{2} \varepsilon\right), & 1+N^{2} \delta
\end{array}\right)
$$

con $\alpha, \beta, \delta$ interi razionali.

Puó evidentemente supporsi $c_{1}-c_{2} \neq 0$, perchè ove si abbia $c_{1}-c_{2}=0$ cioè $c_{1}=c_{2}$ per l'ipotesi fatta che $c_{1}$ e $c_{2}$ siano primi tra loro, si ha $c_{1}=c_{2}= \pm 1$ e perciò $c_{1}+c_{2} \varepsilon= \pm \varepsilon^{2}$, e la trasformata della sostituzione $\Omega$

$$
\left(\begin{array}{ll}
1, & \pm \alpha \varepsilon \\
0, & 1
\end{array}\right) \Omega\left(\begin{array}{cc}
1, & \mp \alpha \varepsilon \\
0, & 1
\end{array}\right)=\left(\begin{array}{cc}
1, & N \beta \\
\pm N \varepsilon^{2}, & 1 \mp N^{2} \beta \varepsilon^{2}
\end{array}\right)=\left(\begin{array}{cc}
1, & N \beta \\
0, & 1
\end{array}\right)\left(\begin{array}{cc}
1, & 0 \\
\pm N \varepsilon^{2}, & 1
\end{array}\right)
$$

è un prodotto di sostituzioni paraboliche.

Supposto allora $c_{1}$ e $c_{2}$ primi tra loro, $c_{1}-c_{2} \neq 0$, si possono determinare gli interi $x_{1}$ e $x_{2}, y_{1}$ e $y_{2}$ in guisa che si abbia rispettivamente:

$$
\left(c_{1}-c_{2}\right) x_{2}+c_{2} x_{1}+d_{2}=0, \quad\left(c_{1}-c_{2}\right) y_{2}+c_{2} y_{1}+a_{2}=0
$$

(17) Quì fucciamo nso del teorema di Dirichlet sulla progresaione aritmetica esteso da E. ECH $v$ alle progressioni $a+b x$ con $a$ e $b$ interi di un curpo $K(\theta)$ e con $a$ primo con l'ideale principalo (b). Cfr. E. ECH к : Ueber di L-Functionen und den Divichletschen Primeahlsatz fïr einen beliebigen Zahlkörper. (Nachrichten von der K. Gessellschaft der Wissenschaften zu Göttingen. Math. phys. Klasse, 1917, pp. 299-318) p. 300. 
e la sostituzione

$$
\left(\begin{array}{cc}
1, & N\left(x_{1}+x_{2} \varepsilon\right) \\
0, & 1
\end{array}\right) \Omega\left(\begin{array}{cc}
1, & N\left(y_{1}+y_{2} \varepsilon\right) \\
0, & 1
\end{array}\right)
$$

ha la forma richiesta.

Lemma $5^{9}$. Se $\Omega$ è una sostituzione del gruppo $\Gamma_{\mu_{(n)}}$, si possono determinare due prodotti di sostituzioni paraboliche $\mathrm{S}_{1}, \mathrm{~S}_{2}$ di $\Gamma_{\boldsymbol{H}_{(n)}}$ tali che si abbia :

$$
S_{1} \Omega S_{2}=\left(\begin{array}{cc}
1+N^{2} \alpha, & N\left(1+N^{2} \gamma_{0}\right) \beta \\
N\left(1+N^{2} \gamma\right), & 1+N^{2} \delta
\end{array}\right)
$$

con $\alpha, \beta, \delta$ interi razionali, $\left(\gamma, \gamma_{0}\right.$ interi coniugati $)$.

Infatti per il Lemma precedente ad $\Omega$ può darsi la forma

$$
\left(\begin{array}{cc}
1+N^{2} \alpha, & N\left(c_{1}+c_{2} \varepsilon^{2}\right) \beta \\
N\left(c_{1}+c_{2} \varepsilon\right), & 1+N^{2} \delta
\end{array}\right)
$$

e supporre in essa $1+N^{2} \alpha$ primo con 6 .

Consideriamo il prodotto

$$
\left(\begin{array}{cc}
1+N^{2} \alpha, & N\left(c_{1}+c_{2} \varepsilon^{2}\right) \beta \\
N\left(c_{1}+c_{2} \varepsilon\right), & 1+N^{2} \delta
\end{array}\right)\left(\begin{array}{cc}
1, & 0 \\
N x, & 1
\end{array}\right)=\Omega_{1}
$$

e indicando con $x_{0}$ un intero complesso radice della congruenza

$$
c_{1}+c_{2} \varepsilon+\left(1+N^{2} \alpha\right) x_{0} \equiv 1
$$

$\left(\bmod . N^{2}\right)$

si scelga l' intero $t$ in guisa che il numero:

$$
\pi=c_{1}+c_{2} \varepsilon+x_{0}\left(1+N^{2} \alpha\right)+N^{2}\left(1+N^{2} \alpha\right) t=1+N^{2} \gamma
$$

sia un numero primo $\left({ }^{18}\right)$. Se $\pi$ è di secondo grado (intero razionale), la $\Omega_{\text {}}$ per le osservazioni $1^{a}$ e $2^{n} \dot{e}$ un prodotto di sostituzioni paraboliche; se $\pi \dot{\mathrm{e}}$ di primo grado, perciò colla parte reale e il coefficiente di $\varepsilon$ primi tra loro, nella (9), posto $x=x_{0}+N^{2} t$, possiamo operare come abbiamo indicato nel lemma precedente e ne segue il lemma ora enunciato.

Lemma $6^{\circ}$. Una sostituzione $\Omega=\left(\begin{array}{cc}1+\mathrm{N}^{2} \mathrm{cc}_{0} \alpha, & \mathrm{Nc}_{0} \beta \\ \mathrm{Nc \gamma}, & 1+\mathrm{N}^{2} \mathrm{cc}_{0} \delta\end{array}\right)$ con $\alpha, \beta, \gamma, \delta$ interi razionali, c $e c_{0}$ inter $i$ coniugati $\dot{e}$ un prodotto di sostituzioni paraboliche di $\Gamma_{\mu(\mathbf{n})}\left({ }^{19}\right)$.

(18) Cfr. (17).

(19) Cfr. 10 (A), p. 315. 
Corollario $1^{\circ}$. Se una sostituzione (unimodulare) $\Omega=\left(\begin{array}{cc}1+\mathrm{N}^{2} \alpha, & \mathrm{Nc}_{0} \beta \\ \mathrm{Nc} \gamma, & 1+\mathrm{N}^{2} \delta\end{array}\right)$ $\operatorname{con} \alpha, \beta, \gamma, \delta$ interi assoluti $\dot{e}$ un prodotto di sostituzioni paraboliche di $\Gamma_{\mu_{(\mathrm{N})}}$ anche la sostituzione unimodulave $\Omega_{\mathrm{t}}=\left(\begin{array}{cc}1+\mathrm{N}^{2} \alpha, & \mathrm{Nc}_{0} \beta_{1} \\ \mathrm{Nc}_{1}, & 1+\mathrm{N}^{2} \delta\end{array}\right)$ con $\beta_{1}$ e $\gamma_{1}$ inter $i$ assoluti $\left(\beta_{1} \gamma_{1}=\beta \gamma\right)$ e un prodotto di sostituzioni paraboliche di $\Gamma_{\mu(\mathrm{N})}\left({ }^{20}\right)$.

Corollario $2^{\circ}$. Se la sostituzione $\left(\begin{array}{cc}1+\mathrm{na}, & \mathrm{nc}_{0} \mathrm{R}_{2} \\ \mathrm{ncR}_{1}, & 1+\mathrm{nd}\end{array}\right)$ con $\mathrm{n}, \mathrm{a}, \mathrm{d}, \mathrm{R}_{1}, \mathrm{R}_{\mathrm{z}}$ inter'i assoluti è un prodotto di sustituzioni paraboliche di $\Gamma_{\mu(\mathbf{n})}$, anche la

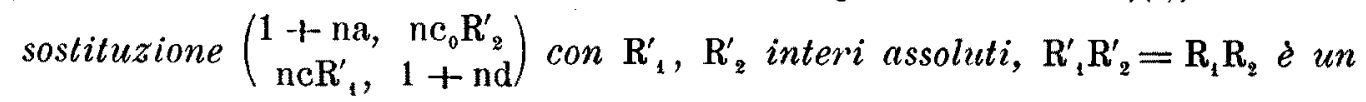
prodotto di sostituzioni paraboliche di $\Gamma_{\mu_{(\mathbf{n})}}\left({ }^{(1)}\right)$.

Lemma $7^{\circ}$. Se $\mathbf{\Omega}$ è una sostituzione del gruppo $\Gamma_{H_{(}(\mathrm{N})}$, la sostitusione $\mathbf{\Omega}^{\circ}$ è un prodotlo di sostituzioni paraboliche di $\Gamma_{\mu(\mathbb{N})}$.

Diremo che una sostituzione $\mathbf{Q}$ appartiene all' esponente $h$, quando $h$ è il più piccolo esponente positivo per il quale si abbia $\Omega^{h}=S$ con $S$ prodotto di sostituzioni paraboliche di $\Gamma_{\mu(N) \text {. }}$

Si puó dimostrare $\left({ }^{(22}\right)$ :

a) se una sostituzione appartiene all' esponente $h$, qualsiasi sua trasformata moltiplicata per un numero finito di sostituzioni paraboliche arbitrarie appartiene all' esponente $h$;

b) data una sostituzione $\boldsymbol{\Omega}$ ad essa se ne può sostituire un' altra

$$
\left(\begin{array}{cc}
1+N^{2} \alpha, & N\left(1+N^{2} \gamma_{0}\right) \beta \\
N\left(1+N^{2} \gamma\right), & 1+N^{2} \delta
\end{array}\right)
$$

con $\alpha, \beta, \delta$ interi razionali, che appartiene allo stesso esponente $h$, ed $h$ deve risultare un divisore dell' indicatore di GaUss

$$
L=\varphi\left[\left\{1+N^{2} \gamma+\left(1+N^{2} \alpha\right)\left(y_{1}+y_{2} \varepsilon\right)\right\}\left\{1+N^{2} \gamma_{0}+\left(1+N^{2} \alpha\right)\left(y_{1}+y_{2} \varepsilon^{2}\right)\right\}\right]
$$

essendo $1+N^{2} \gamma+\left(1+N^{2} \alpha\right)\left(y_{1}+y_{2} \varepsilon\right)$ un numero colla parte reale e il coefficiente di $\varepsilon$ primi tra loro, e $1+y_{1}+y_{2} \varepsilon$ primo con $N$.

Supponiamo come si è detto al lemma $1^{\circ}, N \equiv 0$ (mod. 12) e cominciamo dal provare che se $h$ ha un divisore primo dispari $p$, esso deve essere uguale

( $\left.{ }^{20}\right)$ Cfr. 10 (A), p. 31 s.

(21) Cfr. loc. cit. $\left({ }^{2}\right)$, 11. 2.

(22) Cfr. 10 (A), p. 316. 
a 3 ; per questo dimostriamo che preso un numero primo dispari $p \neq 3$ si possono determinare $y_{1}$ e $y_{2}$ in guisa che $L$ non sia divisibile per $p$.

Si può supporre nella (11), $1+N^{2} \alpha$ primo con $p,\left({ }^{23}\right)$, e siano $x$ ed $a$ due soluzioni delle congruenze

$$
\begin{cases}1+N^{2} \gamma+x\left(1+N^{2} \alpha\right) \equiv 2 & \left(\bmod .9 p^{2}\right) \\ 1+x+9 p^{2} a \equiv 1-\varepsilon & (\bmod .8) .\end{cases}
$$

I numeri $x$ e $a$ esistono, essendo $9 p^{3}$ primo con $1+N^{2} \alpha$, ed 8 primo con $9 p^{2}$. Essendo per le ipotesi fatte il numero $8 \times 9 p^{2}\left(1+N^{2} \alpha\right)$ primo con $1+N^{2} \gamma-1-\left(x+9 p^{2} a\right)\left(1+N^{2} \alpha\right)$ si puó scegliere l'indeterminata $z$ in guisa che il numero

$$
\pi=1+N^{2} \gamma+\left(x+9 p^{2} a\right)\left(1+N^{2} \alpha\right)+8 \times 9 p^{2}\left(1+N^{2} \alpha\right) z
$$

sia primo (24) e poichè non può aversi insieme

$$
\left\{\begin{array}{l} 
\pm 5 \varepsilon^{P} \equiv 2 \\
\pm 5 \varepsilon^{P} \equiv 1-\varepsilon
\end{array}\right.
$$

(mod. 3)

segue che $\pi$ è di primo grado, inoltre possiamo scegliere $z$ in guisa che $\pi$ sia in modulo maggiore di $N^{z}$ e perciò primo coll $N^{2}$.

Si ha poi dalla (13) e dalla prima delle (12)

$$
\pi \pi_{0} \equiv 4
$$

$\left(\bmod .9 p^{2}\right)$

e perciò

$$
\pi \pi_{0}-1 \equiv 3
$$

$\left(\bmod .9 p^{2}\right)$

ossia $L=\varphi\left(\pi \pi_{0}\right)=3+9 p^{2} q$, che non è divisibile per $p$, supposto $p \neq 3$.

Si ha di piủ che la massima potenza di 3 che divide $L$ è il numero 3 stesso, perció $h$ puó contenere come divisore dispari al massimo 3.

Si osservi ancora che dalla (13) e dalla seconda della (12) segue:

$$
\pi \pi_{0} \equiv(1-\varepsilon)\left(1-\varepsilon^{2}\right)=3
$$

(mod. 8)

cioè $\pi \pi_{0}-1=8 l+2$ ovvero $L=2(4 l+1)$ quindi se $h$ è pari la massima potenza del 2 contenuto in esso è 2 , cioè $h$ è un divisore di 6 e perciò $\Omega$ puó appartenere ad un esponente divisore di 6 , ossia $\mathbf{Q}^{6}$ è un prodotto di sostituzioni paraboliche di $\Gamma_{\mu(N)}$.

( $\left.{ }^{23}\right)$ Cfr. 10 (A), P. 318.

(74) Cfr. (17). 
Lemma 8. Se nella sostituzione $\mathrm{Q}=\left(\begin{array}{cc}1+\mathrm{n}^{2} \mathrm{a}, & \mathrm{nb} \\ \mathrm{nc}, & 1+\mathrm{n}^{2} \mathrm{~d}\end{array}\right)$ si ha

$$
\left\{\begin{aligned}
1+n^{2} a \equiv \pm \varepsilon^{?} & & (\bmod . c) ; \rho=0,1,2 ; \\
c \equiv 1 & & \left(\bmod . n^{2}\right)
\end{aligned}\right.
$$

la $\Omega$ es un prodotto di sostituzioni paraboliche di $\Gamma_{\mu(n)}\left({ }^{25}\right)$.

8. Teorema. La sostituzione $\Omega=\left(\begin{array}{cc}1+n \mathrm{a}, & \mathrm{nb} \\ n \mathrm{c}, & 1+\mathrm{nd}\end{array}\right)$ quando il modulo $\mathrm{n}$ è primo con $2(1-\varepsilon)$ è un prodotto di sostituzioni paraboliche di $\boldsymbol{\Gamma}_{\mu(n)}$.

Infatti applicando la trasformazione indicata nel Lemma $1^{\circ}$ e $5^{\circ}$, la può assumere la forma:

$$
\Omega=\left(\begin{array}{cc}
1+N^{2} \alpha, & N\left(1+N^{2} \gamma_{0}\right) \beta \\
N\left(1+N^{2} \gamma\right), & 1+N^{2} \delta
\end{array}\right)
$$

con $N$ primo con $2(1-\varepsilon), \alpha, \beta, \delta$ interi razionali.

E lecito supporre $1+N^{2} \gamma$ numero primo, e

$$
1+N^{2} \gamma \equiv 2+9 \varepsilon
$$

(mod. 36),

e perciò complesso di $1^{\circ}$ grado. Ove ciò non sia si puó trasformare la (14) in guisa che restino soddisfatte le condizioni ora dette. Infatti nella (14) possiamo supporre $1+N^{2} \alpha$ primo con $2(1-\varepsilon)$, [perchè in caso opposto nella sostituzione

$$
\Omega\left(\begin{array}{cc}
1, N\left(1+N^{2} \gamma_{0}\right) x \\
1
\end{array}\right)=\left(\begin{array}{cc}
1+N^{2} \alpha+N^{2}\left(1+N^{2} \gamma\right)\left(1+N^{2} \gamma_{0}\right) x, & N \beta_{1}\left(1+N^{2} \gamma_{0}\right) \\
N\left(1+N^{2} \gamma\right) & 1+N^{2} \delta
\end{array}\right)
$$

essendo $N\left(1+N^{2} \gamma\right)\left(1+N^{2} \gamma_{11}\right)$ primo con $1+N^{2} \alpha$, si puó scegliere $x$ in guisa che il primo coefficiente sia primo con $2(1-\varepsilon)$; basta porre $x$ uguale al prodotto dei fattori (o al fattore) di $2(1-\varepsilon)$ non contenuti in $1+N^{*} \alpha$ ]. Esiste allora una radice $x_{0}$. della congruenza:

$$
1+N^{2} \gamma+\left(1+N^{2} \alpha\right) N^{2} x_{0} \equiv 2+9 \varepsilon
$$

(mod. 36),

(perchè $N^{2}\left(1+N^{2} \alpha\right)$ è primo con $\left.2(1-\varepsilon)\right)$ e si può ancora scegliere l'indeterminata $t$ in guisa che il uumero

$$
\pi=1+N^{2} \gamma+\left(1+N^{\circ} \alpha\right) N^{2} x_{0}+36\left(1+N^{2} \alpha\right) N^{2} t
$$

sia primo complesso [perchè $36\left(1+N^{2} \alpha\right) N^{2}$ è primo con $1+N^{2} \gamma+\left(1+N^{2} \alpha\right) N^{2} x_{0}$ ] e di conseguenza di primo grado a causa della (16).

(25) Cfr. 10 (A), p. 319 
Nella sostituzione

$$
\Omega\left(\begin{array}{cc}
1, & 0 \\
N^{3}\left(x_{0}+36 t,\right. & 1
\end{array}\right)=\left(\begin{array}{cc}
1+N^{2} \alpha, & N \beta_{1} \\
N \pi, & 1+N^{2} \delta_{1}
\end{array}\right)
$$

il fattore primo $\pi$ del $3^{n}$ coefficiente sorldisfa alle due condizioni

$$
\begin{aligned}
& \pi \equiv 1, \\
& \pi \equiv 2+9 \varepsilon
\end{aligned}
$$

e col procedimento del Lemma $6^{\circ}$ potremo formare la sostituzione

$$
S_{1} \Omega S_{2}=\left(\begin{array}{cc}
1+N^{2} \alpha, & N \pi_{0} \beta^{\prime} \\
N \beta & 1+N^{2} \delta^{\prime}
\end{array}\right)
$$

con $S_{1}$ e $S_{2}$ prodotti di sostituzioni paraboliche, $\alpha, \beta^{\prime}, \delta^{\prime}$ interi razionali. Supponiamo senz' altro che nella (14) sia $1+N^{2} \gamma$ primo di primo grado e sia soddisfatta la (15).

Dalla (15) segue

$$
\left(1+N^{2} \gamma\right)\left(1+N^{2} \gamma_{0}\right) \equiv 67
$$

(mod. 36),

perciò $\frac{1}{6} \varphi\left[\left(1+N^{2} \gamma\right)\left(1+N^{2} \gamma_{0}\right)\right]=11+6 q$, ossia il numero $\frac{1}{6} \varphi\left[\left(1+N^{2} \gamma\right)\left(1+N^{2} \gamma_{0}\right)\right]$ è primo con 6 .

Dalla (14) si ha poi

$$
\Omega^{\frac{1}{6} \varphi}=\left(\begin{array}{cc}
\left(1+N^{2} \alpha\right)^{\frac{1}{6} \varphi}+N^{2}\left(1+N^{2} \gamma\right)\left(1+N^{2} \gamma_{0}\right) \alpha_{1}, & N\left(1+N^{2} \gamma_{0}\right) \beta_{1} \\
N\left(1+N^{2} \gamma\right) \gamma_{1}, & \left(1+N^{2} \delta\right)^{\frac{1}{6} \varphi}+N^{2}\left(1+N^{2} \gamma\right)\left(1+N^{0} \gamma_{0}\right) \delta_{1}
\end{array}\right),
$$

$\operatorname{con} \alpha_{1}, \beta_{1}, \gamma_{1}, \delta_{1}$ interi razionali.

Ora avendosi

$$
\begin{array}{lr}
\left(1+N^{2} \alpha\right)^{\frac{1}{6} \varphi} \equiv \pm \varepsilon^{p} & \left(\bmod .1+N^{2} \gamma\right) \\
1+N^{2} \gamma=1 & \left(\bmod N^{2}\right)
\end{array}
$$

per il Lemma $8^{\circ}$ la sostituzione:

$$
\left(\begin{array}{cc}
\left(1+N^{2} \alpha\right)^{\frac{1}{6} \varphi}+N^{2}\left(1+N^{2} \gamma\right)\left(1+N^{2} \gamma_{0}\right) \alpha_{1}, & N\left(1+N^{2} \gamma_{0}\right) \beta_{1} \gamma_{1} \\
N\left(1+N^{2} \gamma\right), & \left(1+N^{2} \delta\right)^{\frac{1}{6} \varphi}{ }^{\frac{1}{6}}+N^{2}\left(1+N^{2} \gamma\right)\left(1+N^{2} \gamma_{0}\right) \hat{\sigma}_{1}
\end{array}\right)
$$

è un prodotto di sostituzioni paraboliche di $\Gamma_{\mu_{(N)}}$, e per il corollario $1^{\circ}$ del 
Lemma $6^{\circ}$ anche la sostituzione ${\Omega^{\frac{1}{\mathbf{b}^{\varphi}}}}=\mathbf{Q}^{11+6 q}$ è un prodotto di sostituzioni paraboliche di $\Gamma_{\mu_{(N)}}$, ossia $\Omega$ appartiene ad un esponente divisore di $11+6 q$ e per il Lemma $7^{\circ}$ all' esponente 1 , e perciò la sostituzione $\Omega=\left(\begin{array}{cc}1+n a, & n b \\ n c, & 1+n d\end{array}\right)$ con $n$ primo con $2(1-\varepsilon)$ è un prodotto di sostituzioni paraboliche di $\Gamma_{\mu_{(n)}}$.

\section{§ 6. Il teorema fondamentale \\ per 1 moduli $m(1-\varepsilon), 3 m, 2 m, 2 m(1-\varepsilon), 6 m$.}

9. Teorema $1^{\circ}$. La sostituzione unimodulare $\Omega=\left(\begin{array}{cc}1+2 \mathrm{~m} \alpha, & 2 \mathrm{~m} \beta \\ 2 \mathrm{~m} \gamma, & 1+2 \mathrm{~m} \delta\end{array}\right)$ con $\mathrm{m}$ dispari, ha il periodo 1 o 3.

Possiamo supporre $\gamma$ primo cun 2 , in caso opposto si può sostituire alla $\mathbf{\Omega}$ l' altra sostituzione $\Omega\left(\begin{array}{cc}1, & 0 \\ 2 m, & 1\end{array}\right)=\left(\begin{array}{cc}1+2 m \alpha, & 2 m \beta \\ 2 m[\gamma+(1+2 m \alpha)], & 1+2 m \delta_{1}\end{array}\right)$ e il numero $\gamma+(1+2 m \alpha)$ è primo con 2 . Indichi ora $x$ una soluzione della congruenza

$$
1+2 m \alpha+2 m \gamma x \equiv 1+6 \varepsilon
$$

(mod. 12),

( $x$ esiste perchè $2 n \gamma$ e 12 hanno per massimo comune divisore $2(1-\varepsilon)^{\lambda}$ $\operatorname{con} \lambda \leq 2)$ e si prenda $t$ in guisa che il numero $\pi=1+2 m \alpha+2 m \gamma x+12 m \gamma t$ sia primo (ció è possibile perchè $12 m \gamma$ è primo con $1+2 m \alpha+2 m \gamma x$ ) e perciò per la (17) di primo grado (è impossibile infatti la congruenza $\left.\pm 5 \varepsilon^{\circ} \equiv 1(\bmod , 6)\right)$; si avrà dalla $(17)$ che il numero primo $p=\pi \pi_{0}$ ha la forma $4 h+3$ cioè $p \equiv 3(\bmod .4)$, e quindi $\left(\frac{-1}{p}\right)=-1 \cdot\left({ }^{2 h}\right)$.

Ora si ha per la trasformata $\boldsymbol{\Omega}_{1}$ di $\boldsymbol{Q}$ (che ha lo stesso periodo di $\boldsymbol{Q}$ )

$$
\mathbf{\Omega}_{1}=\left(\begin{array}{cc}
1, & -(x+6 t) \\
0, & 1
\end{array}\right) \mathbf{\Omega}\left(\begin{array}{cc}
1, & x+6 t \\
0, & 1
\end{array}\right)=\left(\begin{array}{cc}
\pi, & 2 m b \\
2 m \gamma, & 1+2 m d
\end{array}\right)
$$

e sono possibili due casi $\left[\frac{\gamma}{\pi}\right]=1,\left[\frac{\gamma}{\pi}\right]=-1\left({ }^{27}\right)$.

(26) Cfr. Dirichlet-Dhomknd : Lezioni sulla Teoria dei Numeri (traduzione italiana di. A. FAIFOFER) p. 86.

${ }^{(27)}$ Per il significato del simbolo $\left[\frac{\gamma}{\alpha}\right]$ relativo ai corpi quadratici in un corpo algebrico e la sua riduzione all'ordiuario simbolo di LEgEnder, efr. L. BIANCHI, loc. cit. $\left({ }^{6}\right)$, p. 328 a 345. Per il teorema di reciprocita relativo a questi simboli, cfr. E. EcHe: Vorlesungen übev 
$1 .^{\circ}$ Caso. Sia $\left[\frac{\gamma}{\pi}\right]=1$; esisterà (essendo $\pi$ di primo grado), un intero razionale $g$ per il quale si abbia $g^{2} \equiv \gamma$ (mod. $\pi$ ); e sia ancora $a$ un intero razionale tale che si abbia $1+2 m a \equiv g^{-1}\left(\bmod . \pi \pi_{0}\right)$; si avrà

$$
(1+2 n a)^{2} \gamma \equiv 1
$$

$(\bmod . \pi)$,

ovvero

$$
(1+2 m a)^{*} \gamma=1+\pi q
$$

Considerando una sostituzione unimodulare $\Omega_{2}=\left(\begin{array}{cc}1+2 m a)^{2}, & 2 m b_{1} \\ 2 m q & 1+2 m d_{1}\end{array}\right)$ si ha:

$$
\mathrm{Q}_{2} \mathrm{\Omega}_{1}^{-1}=\left(\begin{array}{cc}
(1+2 m a)^{2}, & 2 m b_{1} \\
2 m b, & 1+2 m d_{1}
\end{array}\right)\left(\begin{array}{cc}
1+2 m d, & -2 m b \\
-2 m \gamma, & \pi
\end{array}\right)=\left(\begin{array}{cc}
1+2 m a_{2}, & 2 m b_{2} \\
-2 m, & 1+2 m d_{2}
\end{array}\right)
$$

ed essendo $\left(\begin{array}{cc}1+2 m a_{2}, & 2 m b_{2} \\ -2 m, & 1+2 m d_{2}\end{array}\right)$ un prodotto di sostituzioni paraboliche perchè si ha:

$\left(\begin{array}{cc}1,-a_{2} \\ 0, & 1\end{array}\right)\left(\begin{array}{cc}1+2 m a_{2}, & 2 m b_{2} \\ -2 m, & 1+2 m d_{2}\end{array}\right)\left(\begin{array}{cc}1, & a_{2} \\ 0, & 1\end{array}\right)=\left(\begin{array}{cc}1 & 2 m B \\ -2 m, & 1-4 m B\end{array}\right)=\left(\begin{array}{cc}1,2 m B \\ 0, & 1\end{array}\right)\left(\begin{array}{cc}1, & 0 \\ -2 m, & 1\end{array}\right)$

è $\boldsymbol{Q}_{z} \boldsymbol{Q}_{1}^{-1}=S^{-1}$ ove $S^{-1}$ è un prodotto di sostituzioni paraboliche, ed infine $\Omega_{1}=S \Omega_{2}$ quindi $\Omega_{1}$ (e percio $\left.\Omega\right)$ hanno lo stesso periodo di $\Omega_{2}$.

Consideriamo ora una sostituzione unimodulare $\Omega_{3}=\left(\begin{array}{cc}1+2 m a, & 2 m q_{0} b_{3} \\ 2 m q & 1+2 m d_{3}\end{array}\right)$ con $b_{3}$ e $d_{3}$ interi razionali : è $\Omega_{3}^{2}=\left(\begin{array}{cc}(1+2 m a)^{2}+4 m m^{2} q q_{0} a_{4}, & 2 m q_{0} b_{4} \\ 2 m q c_{4}, & 1+2 m d_{4}\end{array}\right)$ ed essa ha il periodo 1 o 3 ; ha anche il periodo 1 o 3 la sostituzione

$$
\Omega_{4}=\left(\begin{array}{cc}
(1+2 m a)^{2}+4 m^{2} q q_{0} a_{4}, & 2 m q_{0} b_{4} c_{4} \\
2 m q, & 1+2 m d_{4}
\end{array}\right)
$$

dic Theorie der algebraischen Zahlen. (Leiprig 1923, Akademische Verlagsgesellschaft) p. 242 a 249. Il teorema di reciprocità nei corpi quadratici immaginari ha la forma semplicissima: Fra due numeri dispari $\alpha, \beta$ di cui uno almeno sin primario (ciò̀ congruente col quadrato di un numero del corpo rispetto al modulo 4) ha luogo la relazione $\left[\begin{array}{l}x \\ \beta\end{array}\right]=\left[\begin{array}{l}\beta \\ \alpha\end{array}\right]$ (dal teor. 165, paus. 246). So uno dei numeri è pari, si ha l'altro teorema: St $x$ è un numero dispari residuo quadratico del modulo 8 , è $\left[\frac{2}{x}\right]=1$ (dal teorema 167, p. 249). 
poichè il prodotto $Q_{3}^{2} Q_{4}^{-1}$ ha la forma

$$
\left(\begin{array}{cc}
1+4 m^{2} q q_{0} A, & 2 m q_{0} B \\
2 m q C, & 1+4 m^{2} q q_{0} D
\end{array}\right) \quad(A, B, C, D \text { interi assoluti })
$$

ed è quindi (per il Lemma $6^{\circ}, \S 5$ ) un prodotto di sostituzioni paraboliche; ha ancora il periodo 1 o 3 la trasformata di $\Omega_{4}$

$$
\left(\begin{array}{c}
1,2 m q_{0} a_{4} \\
1
\end{array}\right) \Omega_{4}\left(\begin{array}{cc}
1, & -2 m q_{9} a_{4} \\
0, & 1
\end{array}\right)=\left(\begin{array}{cc}
(1+2 m a)^{2}, & 2 m q_{0} B_{4} \\
2 m q, & 1+2 m D_{4}
\end{array}\right)
$$

e infine tenuto conto che questa differisce da $\Omega_{2}$ per una sostituzione parabolica (perchè hanno il primo ed il terzo coefficiente eguali) anche la $\boldsymbol{\Omega}_{2}$ (e perció la $\Omega$ ) ha il periodo 1 o 3 .

c. d. d.

2. Caso. Sia nella $\Omega_{1}=\left(\begin{array}{cc}\pi, & 2 m q \\ 2 m \gamma & 1+2 m d\end{array}\right),\left(\frac{\gamma}{\pi}\right)=-1$. 安 anche

$$
\left[\frac{-\gamma}{\pi}\right]=\left[\frac{-1}{\pi}\right]\left[\frac{\gamma}{\pi}\right]=-\left[\frac{-1}{\pi}\right]=-\left(\frac{-1}{\pi \pi_{0}}\right)=-\left(\frac{-1}{p}\right)=1
$$

quindi la sostituzione $\left(\begin{array}{cc}\pi, & -2 m b \\ -2 m \gamma, & 1+2 m d\end{array}\right)$ per il caso precedente appartiene all' esponente 1 o 3 ; ed anche la $\Omega_{1}$ (ragionando come all' osservazione $2^{\mathrm{a}}, \S 5$ ), ed infine la $\boldsymbol{\Omega}$ ha il periodo 1 o 3 .

10. Teorema $2^{\circ}$. Qualsiase sostiluzione $\left(\begin{array}{cc}1+6 \mathrm{~m} \alpha, & 1+6 \mathrm{~m} \delta \\ 6 \mathrm{~m} \gamma & 6 \mathrm{~m} \beta\end{array}\right)$ con $\mathrm{m}$ primo con 6 si puo esprimere con un prodotto di sostituzioni paraboliche di $\boldsymbol{\Gamma}_{\mu(6 m)}$.

Possiamo supporre (Lemma $\left.1^{\circ}, \S 5\right) m$ intero razionale dispari, primo con 3. Tenuto conto del teorema precedente e ragionando come nella seconda delle Memorie citate dall'A. $\left({ }^{28}\right)$ si prova poi :

a) La sostituzione $\left(\begin{array}{cc}(1+6 m a)^{3}, & 6 m b \\ 6 m c, & 1+6 m d\end{array}\right)$ è un prodotto di sostituzioni paraboliche di $\Gamma_{\mu(6 m)}$.

b) Indicando col simbolo $\left[\frac{c}{1+6 m a}\right]_{3}$ il simbolo generalizzato di JACOBI sui

(28) Cfr. nota (2). 
residui cubici $\left({ }^{29}\right)$, si ha che la sostituzione: $\left(\begin{array}{cc}1+6 m a, & 6 m b \\ 6 m c & 1+6 m d\end{array}\right)$ con $1+6 m a$ primo, di primo grado e $\left[\frac{c}{1+6 m a}\right]_{3}=1$ è un prodotto di sostituzioni paraboliche di $\Gamma_{\mu(\{m)}\left({ }^{30}\right)$.

c) La sostitusione $\mathbf{Q}=\left(\begin{array}{cc}1+6 \mathrm{ma}, & 6 \mathrm{mb} \\ 6 \mathrm{mc}, & 1+6 \mathrm{md}\end{array}\right)$ e un modotto di sostitusioni paraboliche del greuppo $\Gamma_{\mu(i, m)}$.

Infatti possiamo alla $\Omega$ sostituire una sua trasformala, in cui sia $1+6 m a$ primo, di primo grado, e inoltre $a \equiv \varepsilon(\bmod .3)$. Ove questo non sia nella $\Omega$

(29) Cfr. per i residui cubici, P. Bachmann: Die Lehre von dev Kreisteilung und Ihre Berziehungen zur Zahlentheorie. (Keipzig 1872), p. 185 a 199 \& p. 224.

Abbiamo usato per i residui cubici il simbolo $\left[\frac{m}{n}\right]_{3}$ per distinguerlo da quello dei residni qualratici. Per facilita del lettore richimiamo qui le proprietà di questo simbolo di cui furemo uso.

Se $m$ è un numero primo, ed $n$ è primo con $m$, si ha sempre $n^{\frac{N(n)-1}{3}} \equiv s^{P}$ (mod, $n$ ), e si porrà per detinizione $\left[\frac{n}{m}\right]_{3}=\Im^{P}$. La condizione necesararia e nuflicionte perchè sia risolubile la congruenza $x^{3}=n$ (mod. $m$ ) de che si abbia $\left[\frac{n}{m}\right]_{3}=1$. Per i simboli $\left[\frac{n}{m}\right]_{3}$ valgono le seguenti proprietà :

a) $\left[\frac{n}{m}\right]_{3}\left[\frac{n^{\prime}}{m}\right]_{3}=\left[\frac{m n^{\prime}}{m}\right]_{3}$

b) $\left[\frac{-1}{m}\right]_{3}=1,\left[\frac{\varepsilon}{m}\right]=\varepsilon^{N(m)-1}$;

c) $\left[\frac{n}{q}\right]=1$ per $q$ primo intero razionale;

d) $\left[\begin{array}{c}1-\varepsilon \\ a+b \Xi\end{array}\right]=\varepsilon^{\frac{2}{3}(a+1)}$ supposto $a+b \varepsilon$ scritto sotto forma primaria, ciò con $a=-1$ (mod. 3$), b=0$ (mod. 3$)$.

e) Se $m$ e $n$ sone due numeri primi sotto forma primaria (diversi dall'unità) è $\left[\frac{n}{m}\right]_{3}=-\left[\frac{m}{n}\right]_{3}$ (teorema di reciprocita, valido anche quando uno dei due numeri $m$ od $n$ sia il 2 ). Al simbolo generalizkato di $J_{A} \operatorname{coB}\left[\frac{m}{n}\right]_{3}$, con $m$ e $n$ primi tra loro daremo il solito significato; notiamo che si può provare, che se il numero a ha la forma primaria, si ha $\left[\begin{array}{l}\varepsilon \\ x\end{array}\right]_{3}=1, \varepsilon, \varepsilon^{2}$ secondo che si abbia $x x_{0} \equiv 1,4,7$ (mod. 9). Esse infatti si verificano immediatamente per $\alpha$ primo, e con procedimento d'induzione si provano qualunque sia il numero dei fattori in cui si llecompone $\alpha$.

$\left({ }^{30}\right)$ Cfr. per la dimostrazione il teorema precedente. 
si puó supporre sia $c$ primo con 3 , basterà in caso opposto considerare la sostituzione:

$$
\mathbf{Q}\left(\begin{array}{ll}
1, & 0 \\
6 m, & 1
\end{array}\right)=\left(\begin{array}{cc}
1+6 m a, & 6 m b \\
6 m[c+-(1+6 m a)], & 1+6 m d
\end{array}\right)
$$

e se $x$ indica una soluzione della congruenza $a-c x \equiv \varepsilon$ (mod. 3 ), ove si scelga $t$ in guisa che sia il numero $\pi=1+6 m(a-c x)+18 m c t$ primo (e perciò di primo grado) si ha che la trasformata $\Omega_{1}$ di $\Omega$

$$
\Omega_{1}=\left(\begin{array}{cc}
1, x-3 t \\
0, & 1
\end{array}\right) \Omega\left(\begin{array}{cc}
1, & -x+3 t \\
0, & 1
\end{array}\right)=\left(\begin{array}{cc}
\pi, & 6 m b_{1} \\
6 m c, 1+6 m d_{1}
\end{array}\right)
$$

soddisfa alle condizioni richieste.

Ora se è $\left[\frac{c}{\pi}\right]_{3}=1, \Omega_{1}$ e perciò $\Omega$ è un prodotto di sostituzioni paraboliche (caso $b$ ); se invece è $\left[\frac{c}{\pi}\right]_{3}=\varepsilon^{\lambda}$ avendosi

$$
\pi \pi_{0}=(1+6 m \varepsilon+9 q)\left(1+6 m \varepsilon^{2}+9 q_{0}\right)=1-6 m+9 Q
$$

è $\rho=\frac{1}{3}\left(\pi \pi_{0}-1\right)=-2 m+3 Q$ cioè $\rho$ è primo con 3 e se $\rho_{1}$ indica una soluzione della congruenza $\rho_{p_{1}}+\lambda \equiv 0(\bmod .3)$ si ha:

$$
\left[\frac{c \varepsilon^{P_{1}}}{\pi}\right]_{3}=\varepsilon^{\lambda}\left[\frac{\varepsilon}{\pi}\right]^{\rho_{1}}=\varepsilon^{\lambda+p p_{1}}=1
$$

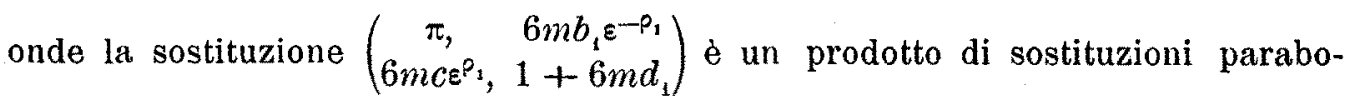
liche di $\Gamma_{\mu(6 m)}$, e quindi $\Omega_{1}$ (per l'osservazione $2^{\mathrm{a}} \S 5$ ) e perciò la $\Omega$ sono prodotti di sostituzion1 paraboliche di $\Gamma_{\mu(6 m)}$.

c. d. d.

Tenuto conto del Lemma $1^{\circ}$ si ha:

Corollario $1^{\circ}$. Tutte le sostituzioni $\left(\begin{array}{cc}1+\mathrm{na}, & \mathrm{nb} \\ \mathrm{nc}, & 1+\mathrm{nd}\end{array}\right)$ quando sia $\mathrm{n}=2^{\lambda}(1-\varepsilon)^{\mu} \mathrm{m}$ con $\mathrm{m}$ primo con $6, \lambda=0,1 ; \mu=0,1$ sono prodotti $d i$ sostituzioni paraboliche di $\Gamma_{\mu(\boldsymbol{n})}$.

Corollario $2^{\circ}$. Il teorema enunciato al $\$ 4$ è vero per $i$ moduli $2^{\lambda}(1-\varepsilon)^{\mu} \mathrm{m}$ con $\mathrm{m}$ primo con $6, \lambda=0,1 ; \mu=0,1$.

Proveremo nel paragrafo seguente che se il modulo $n$ è multiplo di 4 o di $(1-\varepsilon)^{3}$ non è più vero il teorema enunciato nel $\S 4$. 
Qui come applicazione daremo l'interpretazione geometrica dei gruppi

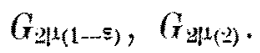

Quando sia $n=1-\varepsilon$ avendosi

$$
(S U)^{2}=\left(\begin{array}{cc}
\varepsilon^{2}, & 0 \\
0, & \varepsilon
\end{array}\right)=1
$$

$(\bmod .1-\varepsilon)$

le (I) del $\$ \$$ diventano:

$$
S^{2}=1, \quad T^{3}=1, \quad U^{2}=1, \quad(S U)^{2}=1, \quad(T U)^{3}=1, \quad\left(S T^{2}\right)^{2}=1,
$$

e le (II) essendo ora $n_{1}=1, n_{2}=--1,(T U)^{2}(T U)^{-2}=1, \quad(T U)^{6}=1$, ed esse sono soddisfatte per le (17).

Le (17) caratteriszano il gruppo ampliato del tetraedro regolare, (di ordine 24). Infatti indicando con $V_{1}, V_{2}, V_{3}, V_{4}$ i vertici di un tetraedro regolare, posto

$$
S=\left(V_{1}, V_{2}\right), \quad T=\left(V_{1}, V_{3}, V_{2}\right), \quad U=\left(V_{1}, V_{2}\right)\left(V_{3}, V_{4}\right)
$$

cioè $S$ uguale alla riflessione sopra il piano che passa per lo spigolo $V_{3} V_{4}$ e il punto medio di $V_{1} V_{2} ; T$ uguale alla rotazione a periodo 3 attorno alla congiungente il vertice $V_{4}$ col centro della faccia $V_{1} V_{2} V_{3} ; U$ uguale al ribaltamento attomo alla retta congiumgente i punti medi degli spigoli opposti $V_{1} V_{2}, V_{3} V_{4}$ i movimenti $S, T, U$ soddisfano alle (17) e generano il gruppo ampliato del tetraedro regolare $\left({ }^{3 t}\right)$, come poteva prevedersi per i resultati del $\$ 1$.

Quando sia $n=2$, le relazioni (I) e (II) del $\& 4$ diventano:

$$
\begin{gathered}
S^{2}=1, \quad T^{3}=1, \quad U^{2}=1, \quad(S U)^{6}=1, \quad(T U)^{3}=1, \quad\left(S T^{2}\right)^{2}=1 \\
{\left[(U S)^{2}(T U)^{2}\right]^{2}=1, \quad\left[(S U)^{2}(T U)^{2}(S U)^{2}\right]^{2}=1 .}
\end{gathered}
$$

L'ultima relazione scritta è conseguenza delle altre. Infatti essa può scriversi :

$$
\begin{gathered}
(S U)^{2}(T U)^{2}(S U)^{2}(S U)^{2}(T U)^{2}(S U)^{2}=(S U)^{2}(T U)^{2}(U S)^{2}(T U)^{2}(S U)^{2}= \\
=(U S)^{2}\left[\left.(U S)^{2}(T U)^{2}\right|^{2}(S U)^{2}=1\right.
\end{gathered}
$$

(31) Cfr. al es. L. Branchr: Lezioni sulla teoria dei gruppi ai sostituzioni e alle equasioni algebriche secondo Gabois. (Pisa, Spoerri 1899), p. 123, p. 129. 
quindi le relazioni fra le $S, T, U$ diventano

$$
\begin{gathered}
S^{2}=1, \quad T^{3}=1, \quad U^{2}=1, \\
(S U)^{6}=1, \quad(T U)^{3}=1, \quad\left(S T^{2}\right)^{2}=1, \quad\left[(U S)^{2}(T U)^{2}\right]^{2}=1 .
\end{gathered}
$$

E facile vedere che il gruppo $G_{2 \mu(2)}$ è isomorfo col gruppo ampliato dell'icosaedro regolare. Infatti indicando con $\infty, 1,2,3,4,5$ un vertice dell' icosaedro e ordinatamente its vertici adiacenti, e con $\infty^{\prime}, 1^{\prime}, 2^{\prime}, 3^{\prime}, 4^{\prime}, 5^{\prime}$ i vertici opposti, posto:

$$
\begin{aligned}
& T=\left(0,4,2^{\prime}\right)\left(0^{\prime}, 4^{\prime}, 2\right)\left(1,3, \infty^{\prime}\right)\left(1^{\prime}, 3^{\prime}, \infty\right) \\
& U=(0, \infty)\left(0^{\prime}, \infty^{\prime}\right)(1,4)\left(1^{\prime}, 4^{\prime}\right)\left(2,2^{\prime}\right)\left(3,3^{\prime}\right), \\
& S=\left(2,4^{\prime}\right)\left(2^{\prime}, 4\right)\left(3, \infty^{\prime}\right)\left(3^{\prime} \infty\right),
\end{aligned}
$$

le $T$ e $U$ rappresentano rispettivamente due rotazioni a periodo 3 e 2 del gruppo ampliato dell'icosaedro e generano un gruppo tetraedrale $G_{12}$ che lascia fermo uno dei cinque ottaedri inscritti nell' icosaedro; $S$ rappresenta una riflessione che trasforma l'ottaedro considerato in un altro ottaedro inscritto, e facilmente si verifica che le $S, T, U$ generano l'intero gruppo dell'icosaedro $\left({ }^{32}\right)$.

Del resto la sostituzione $P=\left(U T^{2}\right) S(T U) S$ è un movimento a periodo 5 attorno ai vertici 1 e $1^{\prime}$, e il gruppo ampliato $G_{12 n}$ dell' jcosaedro si rappresenta per $G_{12}$ con gli indici :

$$
1, \quad P, P^{2}, P^{3}, \quad P^{4}, S, \quad S P, \quad S P^{2}, S P^{3}, S P^{4} .
$$

\section{§ 7. I modull $4 m, 3(1-\varepsilon) m$ come moduli eccezlonall.}

11. Voyliamo ora provare che il teorema enunciato nel $\$ 4$ non sussiste quando il modulo $n$ sia divisibile per il numero 4 , o per il numero $(1-\varepsilon)^{3}$; basterà provare, nei due casi, che esistono delle sostituzioni unimodulari :

$$
\left(\begin{array}{cc}
1+4 m a, & 4 m b \\
4 m c, & 1+4 m d
\end{array}\right),\left(\begin{array}{cc}
1+3 m(1-\varepsilon) a, & 3 m\left(1-\varepsilon^{2}\right) b \\
3 m(1-\varepsilon) c, & 1+3 m(1-\varepsilon) d
\end{array}\right)
$$

che non sono prodotti di sostituzioni paraboliche.

Per il lemma $1^{\circ}$ del $\$ 5$ supponiamo che sia $m$ intero razionale, e limitiamoci ora al primo caso. $\dot{E}$ fondamentale, in vista di quanto ci proponiamo

(32) Cfr. L. Bianchr, loc. eit. $\left({ }^{34}\right)$, p. 124, p. 129. 
di provare, dimostrare il teorema: Tutte le sostituzioni unimodulari,

$$
\left(\begin{array}{cc}
\alpha, & 4 m \beta \\
4 m \gamma & \delta
\end{array}\right), \quad \alpha \equiv \delta \equiv 1 \quad(\bmod , 4 m)
$$

per le quali sia $\left[\frac{\gamma}{\alpha}\right]=1\left({ }^{33}\right)$ formano gruppo.

Siano $\left(\begin{array}{cc}\alpha, & 4 m \beta \\ 4 m \lambda \gamma, & \delta\end{array}\right),\left(\begin{array}{cc}a \lambda, & 4 m b \\ 4 m c, & d\end{array}\right)$ due sostituzioni unimodulari del gruppo $\Gamma_{\mu(1 m)}$ per le quali si abbia:

$$
\left[\frac{\lambda \gamma}{\alpha}\right]=1, \quad\left[\frac{c}{a \lambda}\right]=1
$$

dove con $\lambda$ abbiamo indicato il massimo comun divisore tra il terzo coefficiente della prima sostituzione e il primo della seconda, e dove potremo supporre $a$ e $\lambda$ scelti in guisa che si abbia contemporaneamente:

$$
\left\{\begin{array}{l}
a \equiv 1 \\
\lambda \equiv 1
\end{array} \quad \text { (mod. } 4\right) ; \quad\left\{\begin{array}{l}
a \equiv 1-\varepsilon \\
\lambda \equiv-\left(1-\varepsilon^{2}\right)
\end{array}\right. \text { (mod. 4). }
$$

Sia ancora $\omega$ una radice della congruenza:

$$
a \alpha \omega \equiv 1
$$

(mod. 8)

e possiamo supporre sia $\omega$ primo e primo con $\gamma\left(\right.$ (infatti se $\omega_{0}$ è una radice della congruenza $a \alpha \omega_{0} \equiv 1$ (mod. 8 ), posto $\omega=\omega_{0}+8 q$, si può scegliere $q$ in guisa che il numero $\omega_{0}-1-8 q$ sia primo e primo con $\gamma a$ ).

Avendosi :

$$
\left(\begin{array}{cc}
\alpha, & 4 m \beta \\
4 m \lambda \gamma, & \delta
\end{array}\right)\left(\begin{array}{cc}
a \lambda, & 4 m b \\
4 m c, & d
\end{array}\right)=\left(\begin{array}{cc}
\lambda\left(a \alpha+16 m^{2} b \gamma\right), & 4 m(\lambda a \beta+\delta \delta) \\
4 m(c \alpha+\lambda d \gamma), & d \delta+16 m^{2} c \beta
\end{array}\right)
$$

è

$$
\begin{aligned}
& {\left[\frac{c \alpha+\lambda d \gamma}{\lambda\left(a \alpha+16 m^{2} b \gamma\right)}\right]=\left[\begin{array}{l}
c \\
\lambda
\end{array}\right]\left[\frac{\alpha}{\lambda}\right]\left[\frac{c \alpha+\lambda d \gamma}{a \alpha+16 m^{2} b \gamma}\right]=} \\
& =\left[\frac{c}{\lambda}\right]\left[\begin{array}{l}
\alpha \\
\bar{\lambda}
\end{array}\right]\left[\frac{a c \alpha+\lambda a d \gamma}{a \alpha+16 m^{2} b \gamma}\right]\left[\frac{a}{a \alpha \omega+16 m^{2} b \gamma \omega}\right]\left[\frac{a}{\omega}\right]
\end{aligned}
$$

od anche tenuto conto:

a) $\lambda a d=1+16 m^{2} b c$

b) $\left[\frac{\gamma}{\alpha}|=| \frac{\lambda}{\alpha}\right]$;

(33) Cfr. ("7). 
c) del teorema di reciprocita;

d) $a \alpha \omega \equiv 1(\bmod .8)$ e perciò $\left[\frac{2}{a \alpha \omega}\right]=1$ :

$$
\begin{aligned}
& {\left[\begin{array}{c}
c \alpha+\lambda d \gamma \\
\lambda\left(a \alpha+16 m^{2} b \gamma\right)
\end{array}\right]=\left[\begin{array}{l}
c \\
\lambda
\end{array}\right]\left[\begin{array}{l}
\alpha \\
\lambda
\end{array}\right]\left[\frac{\gamma}{\alpha \alpha+16 m^{2} b \gamma}\right]\left[\frac{b \gamma \omega}{a}\right]\left[\frac{a}{\omega}\right]=}
\end{aligned}
$$

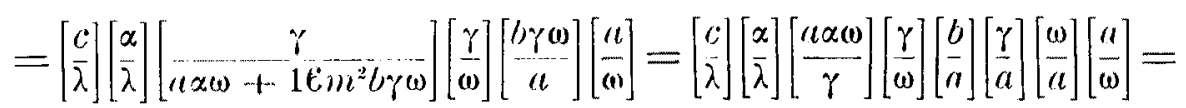

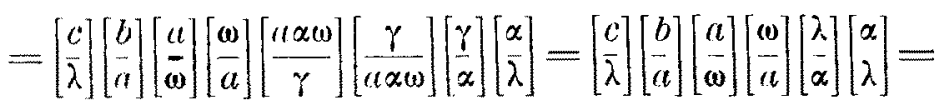

$$
\begin{aligned}
& =\left[\frac{c}{\lambda}\right]\left[\frac{b}{a}\right]\left[\frac{a}{\omega}\right]\left[\frac{\omega}{a}\right] \text {. }
\end{aligned}
$$

Ma è

$$
\left[\frac{b c}{a}\right]=\left[\frac{-1}{a}\right]=\left[\frac{-1}{\iota \alpha \omega}\right]\left[\frac{-1}{\alpha \omega}\right]=\left[\frac{-1}{\alpha \omega}\right]=\left[\frac{-1}{\omega}\right]\left[\frac{-1}{\alpha}\right]=\left[\frac{-1}{\omega}\right]
$$

e perciò $\left[\frac{b}{a}\right]\left[\frac{c}{a} \mid=\left[\frac{-1}{\omega}\right]\right.$, ma è anche $\left[\frac{c}{a}\right]\left[\begin{array}{l}\frac{c}{\lambda} \\ \bar{\lambda}\end{array}\right]=1$, quindi $\left[\frac{b}{a}\right]\left[\begin{array}{l}c \\ \bar{\lambda}\end{array}\right]=\left[\frac{-1}{\omega}\right]$, ed allora $\left[\frac{c \alpha+\lambda d \gamma}{\lambda\left(a \alpha+16 m^{2} b \gamma\right)}\right]=\left[\frac{-1}{\omega}\right]\left[\frac{\alpha}{\omega}\right]\left[\frac{\omega}{a}\right]$.

Distinguiamo ora i due casi $a \equiv 1(\bmod .4), a \equiv 1-\varepsilon(\bmod .4)$ :

a) $a \equiv 1$ (mod. 4). 亡 allora $\left[\frac{a}{\omega}\right]\left[\frac{\omega}{a}\right]=1$, ma è anche $\omega \equiv 1(\bmod .4)$ (cioè $\omega$ è primario) perciò $\left[\frac{-1}{\omega}\right]=1$ ossia $\left[\frac{c \alpha+\lambda d \gamma}{\lambda\left(a \alpha+16 m^{2} b \gamma\right)}\right]=1$.

b) $a \equiv 1-\varepsilon(\bmod .4) ;$ essendo $\alpha \equiv 1(\bmod .4)$ è per la $(18) \omega \equiv-\left(1-\varepsilon^{2}\right)$ (mod. 4), e analogamente se $\omega^{\prime}$ è un altro numero primo, radice della congruenza $(18)$ è $\omega^{\prime} \equiv-\left(1-\varepsilon^{2}\right)(\bmod .4)$ perciò $a \omega^{\prime} \equiv 1, \omega \omega^{\prime} \equiv\left(1-\varepsilon^{2}\right)^{2}(\bmod .4)$ ossia $a \omega^{\prime}$ e $\omega \omega^{\prime}$ sono primari.

Si ha quindi :

$$
\left[\frac{-1}{\omega}\right]\left[\begin{array}{l}
a \\
\omega
\end{array}\right]\left[\frac{\omega}{a}\right]=\left[\frac{-1}{\omega}\right]\left[\frac{\omega^{\prime}}{\omega}\right]\left[\frac{\omega}{\omega^{\prime}}\right]\left[\frac{a \omega^{\prime}}{\omega}\right]\left[\begin{array}{l}
\frac{\omega}{a \omega^{\prime}} \\
]
\end{array}\right]=\left[\frac{-1}{\omega}\right]\left[[ \begin{array} { l } 
{ \omega ^ { \prime } } \\
{ \frac { \omega } { \omega } }
\end{array} ] \left[\begin{array}{l}
\frac{\omega}{\omega^{\prime}} \\
]
\end{array} .\right.\right.
$$

Ora se $\omega\left(\omega^{\prime}\right)$ è intero razionale, anche $\omega^{\prime}=\omega+8 q\left(\omega=\omega^{\prime}+8 q\right)$ si può supporre intero razionale, ed ̀̀

$$
\left[\frac{-1}{\omega}\right]=\left(\frac{1}{\omega}\right)=1,\left[\frac{\omega^{\prime}}{\omega}\right]=\left(\frac{\omega^{\prime 2}}{\omega}\right)=1,\left[\frac{\omega}{\omega^{\prime}}\right]=\left(\frac{\omega^{2}}{\omega^{\prime}}\right)=1,
$$

quindi $\left[\frac{-1}{\omega}\right]\left[\left[\begin{array}{l}\omega^{\prime} \\ \omega\end{array}\right]\left[\begin{array}{l}\omega \\ \omega^{\prime}\end{array}\right]=1\right.$ e perció $\left[\frac{c \lambda+\lambda d \gamma}{\lambda\left(a \alpha+16 m^{2} b_{\gamma}\right.}\right]=1$. 
Siano invece $\omega$ e $\omega^{\prime}$ di $2^{\circ}$ grado possiamo supporli primi con $1-\varepsilon$, e poichè $\omega(1-\varepsilon)$ e $\omega(-3-\varepsilon)$ sono primari si avrà:

a) $\left[\frac{-1}{\omega}\right]=\left(\frac{-1}{\omega \omega_{0}}\right)=-1$, (perchè $\left.\omega \omega_{0} \equiv 3(\bmod .4)\right)$;

b) $\left[\frac{-1}{\omega}\right]\left[\frac{\omega^{\prime}}{\omega}\right]\left[\frac{\omega}{\omega^{\prime}}\right]=-\left[\frac{\omega}{(1-\varepsilon) \omega^{\prime}}\right]\left[\frac{\omega}{1-\varepsilon}\right]\left[\frac{1-\varepsilon}{\omega}\right]\left[\frac{(1-\varepsilon) \omega^{\prime}}{\omega}\right]=-\left[\frac{\omega}{1-\varepsilon}\right]\left[\frac{1-\varepsilon}{\omega}\right]=$

$=-\left[\frac{\omega(-3-\varepsilon)}{1-\varepsilon}\right]\left[\frac{-(3+\varepsilon)}{1-\varepsilon}\right]\left[\frac{1-\varepsilon}{-\omega(3+\varepsilon)}\right]\left[\frac{1-\varepsilon}{-(3+\varepsilon)}\right]=-\left[\frac{-1}{1-\varepsilon}\right]\left[\frac{3+\varepsilon}{1-\varepsilon}\right]\left[\frac{1-\varepsilon}{3+\varepsilon}\right] ;$

c) $\left[\frac{-1}{1-\varepsilon}\right]=\left(\frac{-1}{3}\right)=-1,\left[\frac{3-\varepsilon}{1-\varepsilon}\right]=\left[\frac{\varepsilon}{1-\varepsilon}\right]=1,\left[\frac{1-\varepsilon}{3+\varepsilon}\right]=1 ;\left(\right.$ perchè $(1-\varepsilon)^{n} \equiv 1$ $(\bmod .3+\varepsilon) ;$ percio $\left.\left[\frac{-1}{\omega}\right]\right]\left[\frac{\omega^{\prime}}{\omega}\right]\left[\begin{array}{l}\omega \\ \frac{\omega^{\prime}}{\omega}\end{array}\right]=1$ e quindi $\left[\frac{c \alpha+\lambda d \gamma}{\lambda\left(\alpha \alpha+16 i m^{2} b \gamma\right)}\right]=1$, c. d. d.

Corollario $1^{\circ}$. Se una sostitusione unimodulare

$$
\Omega=\left(\begin{array}{cc}
\alpha, & 4 m \beta \\
4 m \gamma, & \delta
\end{array}\right), \alpha \equiv \delta \equiv 1
$$

e un prodotto di sostituzioni paraboliche del gruppo $\Gamma_{\mu_{(4 m)}}$ e $\left[\frac{\gamma}{\alpha}\right]=+1$.

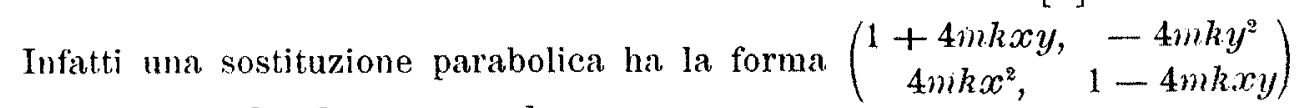
ed è $\left[\frac{k x^{2}}{1+4 m k x y}\right]=\left[\frac{k}{1+4 m k x y}\right]$, ed osservando che se $k$ è pari $1+4 m k x y$ è congruo 1 modulo 8 , è lecito applicare il teorema di reciprocità, e quindi $\left[\frac{k x^{2}}{1+4 m k x y}\right]=1$,

Se poi la $\Omega$ è un prodotto di sostituzioni paraboliche, tenuto conto clel teorema precedente, segue il nostro corollario.

Corollario $2^{\circ}$. Qualunque sostituzione $\Omega=\left(\begin{array}{cc}1+4 \mathrm{ma}, & 4 \mathrm{mb} \\ 4 \mathrm{mc}, & 1+4 \mathrm{md}\end{array}\right)$ con $\left[\frac{\mathrm{c}}{1+4 \mathrm{ma}}\right]=-1$ non può esprimersi come prodotto di sostituzioni paraboliche del gruppo $\mu(\mathbf{4 m})$.

Dalle cose dette ed osservando che si possono costruire quante si vogliono sostituzioni unimodulari, per le quali si abbia $\left[\frac{c}{1+4 m a}\right]=-1$ segue che il teorema del $\$ 4, n^{\circ} 6$ non sussiste per i moduli $4 m$, qualunque sia $m$.

12. La via da seguire nel secondo caso, cioè per i moduli multipli di $(1-\varepsilon)^{3}$ è la medesima, salvo che ai simboli relativi ai residui quadratici sostituiremo i simboli relativi ai residui cubici. 
Teorema. Tutte le sostituzioni unimodulari

$$
\left(\begin{array}{cc}
\alpha, & 3 m\left(1-\varepsilon^{2}\right) \beta \\
3 m(1-\varepsilon) \gamma, & \delta
\end{array}\right) \quad \alpha \equiv \delta \equiv 1 \quad[\bmod .3(1-\varepsilon) m]
$$

per le quali sia $\left[\frac{\gamma}{\alpha}\right]_{3}=1\left({ }^{34}\right)$ formano gruppo.

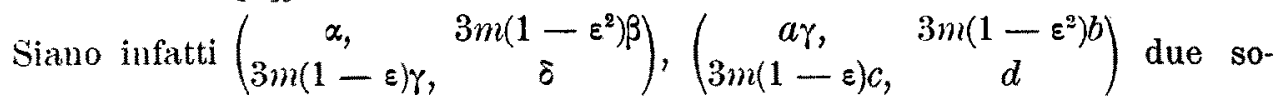
stituzioni di $\Gamma_{\mu|3 m(1-\varepsilon)|}$, cioè tali che si abbia:

$$
\alpha \equiv \delta \equiv a \lambda \equiv d \equiv 1
$$

e inoltre sia $\left[\frac{\gamma \lambda}{\alpha}\right]_{3}=1,\left[\frac{c}{a \lambda}\right]_{3}=1$.

Abbiamo indicato con $\lambda$ il massimo comun divisore tra il terzo coefficiente della prima sostituzione ed il primo della seconda, quindi $l$ ed $a$ sono primi tra loro, e supponiamo, come del resto è lecito $a \equiv \lambda \equiv 1$ (mod. 3 ).

Il prodotto delle due sostituzioni è

$$
\left(\begin{array}{cc}
\lambda\left(a \alpha+27 m^{2} b \gamma\right), & 3 m\left(1-\varepsilon^{2}\right)(a \lambda \beta+b \delta) \\
3 m(1-\varepsilon)(c \alpha+\lambda d \gamma), & d \delta+27 m^{2} c \beta
\end{array}\right)
$$

e occorre provare che $\left[\frac{c \alpha+\lambda d \gamma}{\lambda\left(a \alpha+27 m^{2} b \gamma\right)}\right]_{3}=1$

Tenuto conto che $a c \alpha+\lambda a d \gamma=c\left(a \alpha+27 m^{2} b \gamma\right)+\gamma$ si ha:

$$
\begin{aligned}
& {\left[\frac{c \alpha+\lambda d \gamma}{\lambda\left(a \alpha+27 m^{2} b \gamma\right)}\right]_{3}=\left[\frac{c}{\lambda}\right]_{3}\left[\frac{\alpha}{\lambda}\right]_{3}\left[\frac{c \alpha+\lambda d \gamma}{a \alpha+27 m^{2} b \gamma}\right]_{3}=} \\
& =\left[\frac{c}{\lambda}\right]_{3}\left[\begin{array}{l}
\alpha \\
\lambda
\end{array}\right]_{3}\left[\frac{c \alpha+\lambda d \gamma}{\alpha \alpha+27 m^{2} b \gamma}\right]_{3}\left[\frac{a}{a \alpha+27 m^{2} b \gamma}\right]_{3}^{3}= \\
& =\left[\frac{c}{\lambda}\right]_{3}\left[\frac{\alpha}{\lambda}\right]_{3}\left[\frac{\gamma}{\alpha \alpha+27 m^{2} b \gamma}\right]_{3}\left[\frac{a}{a \alpha+27 m^{2} b \gamma}\right]_{3}^{2} \text {. }
\end{aligned}
$$

Poichè è $a$ primo con $1-\varepsilon$, applicando all'ultimo fattore il teorema di reciprocità, si ha:

$$
\left[\frac{c \alpha+\lambda d \gamma}{\lambda\left(a \alpha+27 m^{2} b \gamma\right)}\right]_{3}=\left[\begin{array}{l}
c \\
\bar{\lambda}
\end{array}\right]_{3}\left[\begin{array}{l}
\lambda \\
\bar{\lambda}
\end{array}\right]_{3}\left[\frac{\gamma}{a \alpha+27 m^{2} b \gamma}\right]_{3}\left[\frac{m^{2} b \gamma}{a}\right]_{3}^{2} .
$$

(34) Cfr. $\left({ }^{29}\right)$. 
Distingueremo ora $\mathrm{i}$ tre casi :

a) $a \equiv 1$ [mod. $3(1-\varepsilon)]$;

b) $a \equiv 4$ [mod. $3(1-\varepsilon)]$

c) $a \equiv-2[\bmod .3(1-\varepsilon)]$.

Caso a). Sia $a \equiv$ [mod. $3(1-\varepsilon)]$; per qualunque $\gamma$ è :

quindi :

$$
\left[\frac{\gamma}{a \alpha+27 m^{2} b \gamma}\right]_{3}=\left[\frac{a \alpha}{\gamma}\right]_{3}=\left[\frac{a}{\gamma}\right]_{3}\left[\frac{\alpha}{\gamma}\right]_{3},
$$

$$
\left[\frac{c \alpha+\lambda d \gamma}{\lambda\left(a \alpha+27 m^{2} b\right)_{Y}}\right]_{3}=\left[\frac{c}{\lambda}\right]_{3}\left[\frac{\alpha}{\lambda}\right]_{3}\left[\frac{a}{\gamma}\right]_{3}\left[\frac{\alpha}{\gamma}\right]_{3}\left[\frac{m^{2}}{a}\right]_{3}^{2}\left[\frac{b}{a}\right]_{3}^{2}\left[\frac{\gamma}{a}\right]_{3}^{2}
$$

Ma avendosi $\left[\frac{\lambda \gamma}{\alpha}\right]_{3}=1$ e $\alpha \equiv 1[\bmod .3(1-\varepsilon)]$ è anche $\left[\frac{\alpha}{\lambda \gamma}\right]_{3}=\left[\frac{\alpha}{\lambda}\right]_{3}\left[\frac{\alpha}{\gamma}\right]_{3}=1 ;$ $\mathrm{da}\left[\frac{c}{a \lambda}\right]_{3}=1$ segue $\left[\frac{c}{\lambda}\right]_{3}=\left[\frac{c}{a}\right]_{3}^{2} ;$ infine è $\left[\frac{a}{\gamma}\right]_{3}=\left[\frac{\gamma}{a}\right]_{3}$ quindi

$$
\left[\frac{c \alpha+\lambda d \gamma}{\lambda\left(a \alpha+27 m^{2} b \gamma\right)}\right]_{3}=\left[\frac{c}{\lambda}\right]_{3}\left[\frac{m^{2}}{a}\right]_{3}^{2}\left[\frac{b}{a}\right]_{3}^{2}=\left[\frac{c}{a}\right]_{3}^{2}\left[\frac{m^{2}}{a}\right]_{3}^{2}\left[\frac{b}{a}\right]_{3}^{2}=\left[\frac{m^{2} b c}{a}\right]_{3}^{2}=\left[\frac{-1}{a}\right]_{3}^{2}=1 .
$$

Caso b). Sia $a \equiv 4[\bmod .3(1-\varepsilon)]$ e quindi $\lambda \equiv 7[\bmod .3(1-\varepsilon)]$; è di conseguenza $a a_{0} \equiv 7(\bmod .9), \lambda \lambda_{0} \equiv 4(\bmod .9)$ ed infine

$$
\left[\frac{\varepsilon}{a}\right]_{3}=\varepsilon^{2}, \quad\left[\frac{\varepsilon}{\lambda}\right]_{3}=\varepsilon\left({ }^{35}\right)
$$

Posto $\gamma=\varepsilon^{p} \Pi(q) \Pi(\omega), \quad a \alpha+27 m^{2} b \gamma=\Pi_{1}(q) \Pi_{1}(\omega)$ ove con $q$ e $\omega$ abbiamo indicato rispettivamente $i$ fattori primi (sotto forma primaria) di primo e secondo grado dei numeri $\gamma$ e $a \alpha+27 m^{2} b \gamma$, osservando che è

si ha:

$$
a \alpha+27 m^{2} b \gamma \equiv 4
$$

$[\bmod 3(1-\varepsilon)]$

$$
\begin{gathered}
{\left[\frac{\gamma}{a \alpha+27 m^{2} b \gamma}\right]_{3}=\left[\frac{\varepsilon}{a \alpha+27 m^{2} b \gamma}\right]_{3}^{P}\left[\frac{\Pi(q) \Pi(\omega)}{\Pi_{1}(q) \Pi_{1}(\omega)}\right]_{3}=\varepsilon^{2 P}\left[\frac{\Pi_{1}(q) \Pi(\omega)}{\Pi(q) \Pi(\omega)}\right]_{3}=} \\
=\varepsilon^{2 P}\left[\frac{a \alpha+27 m^{2} b \gamma}{\gamma}\right]_{3}=\varepsilon^{2 P}\left[\frac{a}{\gamma}\right]_{3}\left[\frac{\alpha}{\gamma}\right]_{3}=\varepsilon^{2 P}\left[\frac{\alpha}{\gamma}\right]_{3}\left[\frac{a}{\varepsilon^{\rho} \Pi(q) \Pi(\omega)}\right]_{3}=\varepsilon^{2 P}\left[\frac{\alpha}{\gamma}\right]_{3}\left[\frac{\Pi(q) \Pi(\omega)}{a}\right]_{3}= \\
=\varepsilon^{2 P}\left[\frac{\alpha}{\gamma}\right]_{3}\left[\frac{\varepsilon^{P} \Pi(q) \Pi(\omega)}{a}\right]_{3}\left[\frac{\varepsilon}{a}\right]_{3}^{2 P}=\varepsilon^{2 P}\left[\frac{\alpha}{\gamma}\right]_{3}\left[\frac{\gamma}{a}\right]_{3} \varepsilon^{4 P}=\left[\frac{\alpha}{\gamma}\right]_{3}\left[\frac{\gamma}{a}\right]_{3} ;
\end{gathered}
$$

e ancora come prima $\left[\frac{c \alpha+\lambda d \gamma}{\lambda\left(a \alpha+27 m^{2} b \gamma\right)}\right]_{3}=1$.

(35) Cfl. $\left({ }^{29}\right)$. 
Caso c). In modo analogo al caso b) si esamina l'ultimo caso:

$$
a \equiv-2 \quad \text { [mod. } 3(1-\varepsilon)] .
$$

Corollario $1^{\circ}$. Se una sostiluzione unimodulare

$$
\Omega=\left(\begin{array}{cc}
\alpha, & 3 m\left(1-\varepsilon^{2}\right) \beta
\end{array}\right), \quad \alpha \equiv \delta \equiv 1
$$

è un prodotto di sostituzioni paraboliche del gruuppo $\Gamma_{\mu[3 m(1-s)]}, e\left[\frac{\gamma}{\alpha}\right]_{3}=1$.

Infatti se $\Omega$ è una sostituzione parabolica, ha la forma

e per essa si ha:

$$
\left(\begin{array}{cc}
1+3 m(1-\varepsilon) k x y, & -3 m(1-\varepsilon) k y^{2} \\
3 m(1-\varepsilon) k x^{2}, & 1-3 m(1-\varepsilon) k x y
\end{array}\right)
$$

$$
\left[\frac{k x^{2}}{1+3 m(1-\varepsilon) k x y}\right]_{3}=\left[\frac{k}{1+3 m(1-\varepsilon) k x y}\right]_{3}\left[\frac{x}{1+3 m(1-\varepsilon) k x y}\right]_{3}^{2} .
$$

Posto $k=\varepsilon^{P} \Pi(q) \Pi(\omega)$, tenuto conto che

si ha:

$$
[1+3 m(1-\varepsilon) k x y]\left[1+3 m\left(1-\varepsilon^{2}\right) k_{0} x_{0} y_{0}\right] \equiv 1
$$

(mod. 9)

$$
\begin{aligned}
{\left[\frac{k}{1+3 m(1-\varepsilon) k x y}\right]_{3} } & =\left[\frac{\varepsilon}{1+3 m(1-\varepsilon) k x y}\right]_{3}^{p}\left[\frac{1+3 m(1-\varepsilon) k x y}{\Pi(q) \Pi(\omega)}\right]_{3}= \\
& =\left[\frac{1+3 m(1-\varepsilon) k x y}{k}\right]_{3}=1
\end{aligned}
$$

analogamente $\left[\frac{x}{1+3 m(1-\varepsilon) k x y}\right]_{3}=1$ e perciò $\left[\frac{k x^{2}}{1+3 m(1-\varepsilon) k x y}\right]_{3}=1$.

Se poi la $\Omega$ è un prodotto di sostituzioni paraboliche, tenuto conto del teorema precedente segue il nostro corollario.

Corollario $2^{\circ}$. Qualunque sostituzione $\left(\begin{array}{cc}1+3 \mathrm{~m}(1-\varepsilon) \mathrm{a}, & 3 \mathrm{~m}\left(1-\varepsilon^{2}\right) \mathrm{b} \\ 3 \mathrm{~m}(1-\varepsilon) \mathrm{c}, & 1+3 \mathrm{~m}(1-\varepsilon) \mathrm{d}\end{array}\right)$ nella quale sia $\left[\frac{\mathrm{c}}{1+3 \mathrm{~m}(1-\varepsilon) \mathrm{a}}\right]_{3}=-1$ non pud esprimersi come prodotto di sostituzioni paraboliche del gruppo $\Gamma_{\mu[3 n(1-\varepsilon)]}$.

Dalle cose dette segue che il teorema del $\S 4, n^{\circ} 6$, non sussiste per i moduli $3(1-\varepsilon) m$, qualunque sia $m\left({ }^{36}\right)$.

Firenze, giugno 1924.

(36) In altra nota ci proponiamo di trovare le alteriori condizioni che caratterizznno

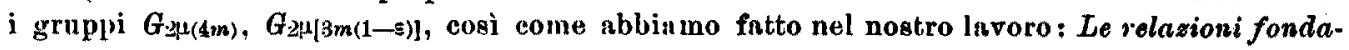
mentali del gruppo modulare finito con coefficiente nel campo di Gauss, (loc. cit. (2)). 